\title{
Characteristic Modeling and Control of Servo Systems with Backlash and Friction
}

\author{
Yifei Wu, Zhihong Wang, Yuanyuan Li, Wei Chen, Renhui Du, and Qingwei Chen \\ School of Automation, Nanjing University of Science and Technology, Nanjing 210094, China \\ Correspondence should be addressed to Yifei Wu; wuyifei0911@163.com
}

Received 23 August 2013; Revised 4 December 2013; Accepted 8 December 2013; Published 19 February 2014

Academic Editor: Bo-Chao Zheng

Copyright (C) 2014 Yifei Wu et al. This is an open access article distributed under the Creative Commons Attribution License, which permits unrestricted use, distribution, and reproduction in any medium, provided the original work is properly cited.

A novel approach for modeling and control of servo systems with backlash and friction is proposed based on the characteristic model. Firstly, to deal with friction-induced nonlinearities, a smooth Stribeck friction model is introduced. The backlash is modeled by a continuous and derivable mathematical function. Secondly, a characteristic model in the form of a second-order slowly timevarying difference equation is established and verified by simulations. Thirdly, a composite controller including the golden-section adaptive control law and the integral control law is designed and the stability of the closed-loop system is analyzed. The simulation and experimental results show that the proposed control scheme is effective and can improve the steady-state precision and the dynamic performance of the servo system with backlash and friction.

\section{Introduction}

Servo systems have been widely used in various applications, such as machine tools, robots, semiconductor manufacturing manipulators, radars, and satellite antennas. With the development of technology, there is an increasing demand for high precision position controllers, which should have fast tracking behavior, disturbance rejection ability, and robustness to uncertainties. However, the nonsmooth nonlinearities including backlash and friction are often present in servo systems, which can lead to steady-state tracking errors, undesired stick-slip motion, and limit cycles $[1,2]$. Although some control approaches [1,2], such as PD control, fuzzy control, adaptive control, robust adaptive control, neural networks control, and sliding mode control, have been given to reduce the backlash and friction effect, it is still a challenging problem because of the nondifferentiable nature of the backlash and friction. In addition, backlash and friction have previously been studied separately $[1,2]$, more rarely together. In many servo systems, a controller designed to compensate only for friction may perform poor performance in the presence of backlash, and vice versa, because they coexist in these systems. Thus, both of them should be taken into consideration in the controller design.
Backlash is present in servo systems with gear transmissions, where the motor temporarily loses direct contact with the load when the backlash gap opens. It may often cause delays, oscillations, and steady-state errors. Since backlash is characterized by nondifferentiable nonlinearity, which is poorly known, the control system with unknown backlash is a difficult problem. Therefore, a number of different approaches to model and compensate for backlash have been investigated for several decades. A recent survey paper [2] summarizes the backlash models and the compensation methods. Different mathematical models have been developed to describe the backlash phenomenon, such as dead-zone model and hysteresis model [2]. The dead-zone model is a static, scalar nonlinear function, which means that it is relatively easy to be analyzed. In this paper, we will adopt it as the backlash model. Many papers deal with the compensation for the backlash. An adaptive control scheme developed by Tao and Kokotović for the systems with unknown backlash is given in [3]. The scheme of Selmic and Lewis [4] implements a dynamic inversion compensation for backlash using the backstepping technique with neural networks. In [5], an optimal control scheme is employed for backlash compensation and a feedback linearization is designed to decouple the multivariable nonlinear dynamics 
so that backlash compensation and tracking control can be both achieved. In [6], a novel adaptive control design is achieved by introducing a smooth inverse function of the dead zone and using it in the controller design with the backstepping technique. A hybrid model based on model predictive control (MPC) scheme for backlash compensation is introduced in [7]. A second-order sliding mode observer ensuring the finite time convergence of estimated state values towards real state values in a nonlinear mechanical system with backlash is given in [8]. A linear estimator for the fast and accurate estimation of the position and velocity in the presence of backlash in automotive power trains is described in [9]. In [10], an adaptive dynamic surface control scheme combined with sliding mode control to compensate for backlash nonlinearities in a linear stage motion system is present. In [11], an extended state observer (ESO) combined with the adaptive sliding mode control theory is proposed to deal with a nonlinear pneumatic servo system characterized with input backlash.

Friction is one of the most important limitations in high precision position systems. It can cause tracking errors, selfexcited vibration, and limit cycles [1]. Friction modeling and compensation have been studied extensively in the past few decades but is still full of interesting problems due to their practical significance and the complex behavior of friction. Satisfactory friction compensation can be obtained if a good friction model is available. However, friction is a highly nonlinear phenomenon, which is difficult to be described by a simple model [1]. Some models, which include both static and dynamic, have been proposed by researchers. A classical static friction model contains the property such as Coulomb friction, stiction, Stribeck effect, and viscous friction [1]. Dynamic friction models, mainly including the Dahl model [12] and the LuGre model [13], have been proposed and shown to be more beneficial. However, experimental measurements have proved that a good static friction model can approximate the real friction force with a degree of $90 \%$ fidelity [14]. The dynamic friction behaviour can be introduced in the static model as a bounded additive model uncertainty [1]. Therefore, the static friction model with Stribeck effect has a great significance for practical applications. The control methods for friction compensation are divided into three categories $[1,15]$ : the model-oriented friction compensation scheme, the adaptive compensation scheme, and the soft computing approach. In [16], a nonlinear proportional-integral-derivative (NPID) control has been designed to improve compensation for friction by applying a state feedback NPID control law with time-varying state feedback gains. In [17-19], a fuzzy system is utilized to adaptively learn unknown friction behavior and compensate for it. In [20], an adaptive friction compensator structure is proposed in which the Stribeck friction term is approximated by RBF-type neural network. To handle model uncertainties, robust adaptive control techniques [21,22] are also very popular for friction compensation.

From the preceding discussion, it can be found that the majority of previous studies have addressed either the friction compensation problem or the backlash compensation problem. Very few papers deal with the control of systems in the presence of backlash and friction [23, 24]. This motivates us to carry out present work. In this paper, we adopt a new adaptive control method based on a characteristic model to handle both friction and backlash. The characteristic modeling theory and methods, proposed by Wu in the 1990s, are an integrated and practical modeling and control theory based on the control-oriented design thought, and improved gradually in the recent 20 years, which has already been applied successfully in more than 400 systems belonging to nine kinds of engineering plants in the field of astronautics and industry [25-27]. The characteristic modeling is based on the dynamics characteristics and control performance requirements of the plants, rather than being only based on accurate plant dynamics analysis. This method provides a feasible low-order intelligent controller design method for various complicated plants with nonlinearities and uncertainties, and whose output variables cannot be measured online continuously. Currently, it has not been applied in servo systems with backlash and friction. Therefore, we will attempt to use a characteristic model to describe the servo system with backlash and friction and design the controller.

The remainder of this paper is organized as follows. In Section 2, we describe the original dynamic model and the characteristic model of the servo system with backlash and friction. In Section 3, the composite controller including the golden-section adaptive control law and the integral control law is designed, and the stability of the closed-loop system is analyzed. Sections 4 and 5 present consecutively the simulation and experimental results. Finally, the conclusion is given in Section 6.

\section{Characteristic Modeling}

The characteristic modeling is based on the dynamics characteristics and control performance requirements of the plants, rather than being only based on accurate plant dynamics analysis. This method provides a feasible low-order intelligent controller design method for various complicated plants with nonlinearities and uncertainties.

A characteristic model has the following features [25].

(1) For the same input, a plant characteristic model is equivalent to its practical plant in output. In a dynamic process, the output error can be maintained within a permitted range. In the steady state, their outputs are equal.

(2) Besides plant characteristics, the form and order of a characteristic model mainly rely on control performance requirements.

(3) The structure of a characteristic model should be simpler, than an original dynamic equation, easier, and more convenient to be realized in engineering.

(4) A characteristic model is different from the reducedorder model of a high-order system. It compresses all the information of the high-order model into several characteristic parameters. In the bandwidth of the control system, no information is lost. In general, 


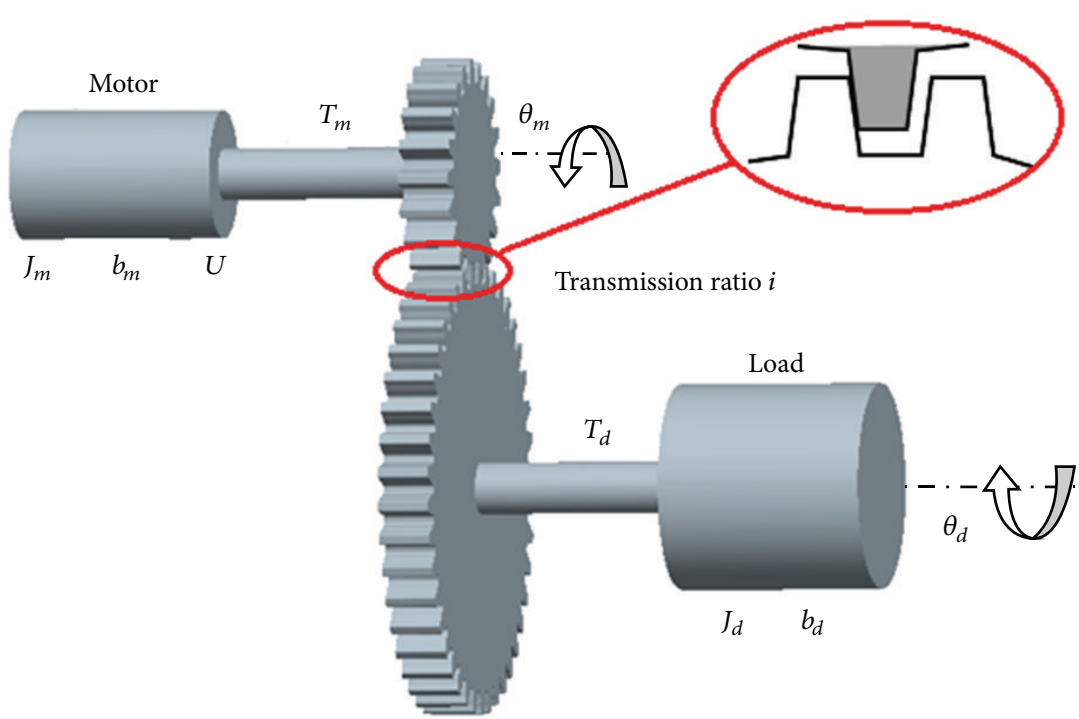

FIGURE 1: Structure of a typical electromechanical system driven by gears.

a characteristic model is represented by a slowly timevarying difference equation.

Characteristic modeling mainly considers the characteristic relation between control input and output. There are two approaches to establish characteristic model. One is to establish the characteristic model directly according to the dynamic features of the practical plant and the control performance requirements. And the other is to establish the characteristic model based on the original dynamic model. The latter one is applied in this paper; that is, the original dynamic model containing backlash and friction seems as the standard plant model of the system. Then the characteristic model is established based on it.

2.1. Dynamic Model. The structure of a typical electromechanical system driven by gears is shown in Figure $1 . \theta_{m}$ and $\dot{\theta}_{m}$ denote the angular displacement and the angular velocity of the motor, respectively. $\theta_{d}$ and $\dot{\theta}_{d}$ denote the angular displacement and the angular velocity of the load, respectively, $J_{m}$ and $J_{d}$ denote the rotational inertia of the motor and the load, respectively, $T_{m}$ and $T_{d}$ denote the torque of the motor and the load, respectively, $b_{m}$ and $b_{d}$ denote the viscous friction coefficient of the motor and the load, respectively, and $i$ denotes the gear ratio.

The dead-zone model is used to model the backlash in Figure 1 , and $\tau_{1}$ can be expressed as

$$
\begin{gathered}
\tau_{1}=k_{b} f(z) \\
f(z)= \begin{cases}z+\alpha & z<\alpha \\
0 & |z| \leq \alpha \\
z-\alpha & z>\alpha,\end{cases}
\end{gathered}
$$

where $\tau_{1}$ denotes the elastic torque between the motor and the load, $k_{b}$ denotes the stiffness, $f(z)$ denotes the deadzone function, $z\left(z=\theta_{m}-i \theta_{d}\right)$ denotes the relative angular displacement between the motor and the load, and $\alpha$ denotes half of the backlash width.

Besides the backlash nonlinearity, the friction nonlinearity should be also considered in the system. The Stribeck model is used to describe the friction in this paper. So the friction torque $\tau_{2}$ can be expressed as

$$
\tau_{2}=\left[F_{c}+\left(F_{s}-F_{c}\right) e^{-\left(\dot{\theta}_{m} / \dot{\theta}_{s}\right)^{\delta}}\right] \operatorname{sgn}\left(\dot{\theta}_{m}\right)+B \dot{\theta}_{m}
$$

where $F_{c}, F_{s}, B, \dot{\theta}_{m}$, and $\dot{\theta}_{s}$ represent the Coulomb friction, the maximum static friction, the viscous damping coefficient, the angular velocity of the motor, and the Stribeck speed, respectively, and $\operatorname{sgn}(\cdot)$ represents the sign function. $\dot{\theta}_{s}$ and $\delta$ are empirical constants, which are usually assumed to be 0.05 and 2 , respectively.

The dynamic equations of a typical electromechanical system driven by gears can be expressed as

$$
\begin{gathered}
U(t)=k_{e} \dot{\theta}_{m}(t)+R I(t)+L \frac{d I(t)}{d t} \\
J_{m} \ddot{\theta}_{m}(t)+b_{m} \dot{\theta}_{m}(t)=T_{m}-\tau_{1}-\tau_{2} \\
J_{d} \ddot{\theta}_{d}(t)+b_{d} \dot{\theta}_{d}(t)=i \tau_{1} \\
T_{m}=k_{d} I(t) \\
\tau_{1}=k_{b} f(z)
\end{gathered}
$$




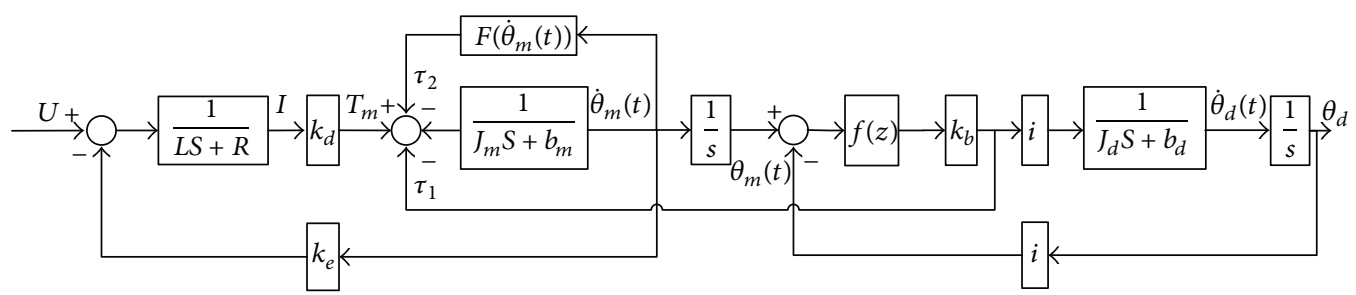

FIGURE 2: Structure diagram of the electromechanical system.

$$
\begin{gathered}
f(z)= \begin{cases}z+\alpha & z<-\alpha \\
0 & |z| \leq \alpha \\
z-\alpha & z>\alpha\end{cases} \\
\tau_{2}=\left[F_{c}+\left(F_{s}-F_{c}\right) e^{-\left(\dot{\theta}_{m} / \dot{\theta}_{s}\right)^{\delta}}\right] \operatorname{sgn}\left(\dot{\theta}_{m}\right)+B \dot{\theta}_{m},
\end{gathered}
$$

where $U(t)$ denotes the armature voltage, $I(t)$ denotes the armature current, $R$ and $L$ denote the resistance and the inductance, respectively, $k_{e}$ denotes the back electromotive force coefficient of the motor, and $k_{d}$ denotes the torque coefficient of the motor.

The system (2.1) can also be represented by the structure diagram as shown in Figure 2.

2.2. Characteristic Model. The nonlinear system is expressed as

$$
\dot{x}(t)=f\left(x, \dot{x}, \ldots, x^{(n)}, u, \dot{u}, \ldots, u^{(m)}\right) .
$$

Let $x=x_{1}, \dot{x}=x_{2}, \ldots, x^{(n)}=x_{n+1}$ and $u=u_{1}, \dot{u}=$ $u_{2}, \ldots, u^{(m)}=u_{m+1}$; then (5) can be rewritten as

$$
\dot{x}(t)=f\left(x_{1}, \ldots, x_{n+1}, u_{1}, \ldots, u_{m+1}\right) .
$$

This nonlinear system (6) is assumed as follows (see [28]).

Assumption 1. The system is a SISO system.

Assumption 2. The order of control input $u$ is 1 .

Assumption 3. If all the variables $x_{i}$ and $u_{i}$ in $f(\cdot)$ are zero, $f(\cdot)=0$.

Assumption 4. $f(\cdot)$ is continuous differentiable to all the variables, and all partial derivatives are bounded.

Assumption 5. $|f(x(t+\Delta t), u(t+\Delta t))|-|f(x(t), u(t))|<M \Delta t$, where $M$ is positive constant and $\Delta t$ is sample period.

Assumption 6. All the variables $x_{i}$ and $u_{i}$ are bounded. This assumption can be satisfied easily in practical engineering.

Lemma 7 (see [28]). If the system (5) satisfies the above Assumptions 1-4 and sample time $\Delta t$ satisfies some certain conditions, the characteristic model of the system can be established in the form of a second-order time-varying difference equation as

$$
\begin{aligned}
x(k+1)= & \alpha_{1}(k) x(k)+\alpha_{2}(k) x(k-1) \\
& +\beta_{0}(k) u(k)+\beta_{1}(k) u(k-1),
\end{aligned}
$$

where $\alpha_{1}(k), \alpha_{2}(k), \beta_{0}(k)$, and $\beta_{1}(k)$ are coefficients.

When the controlled system is stable and satisfies Assumptions 5 and 6, the following conclusions can be drawn.

(1) In a dynamic process, under the same input, the output of the characteristic model is equal to that of the practical plant (suitably selected sampling period $\Delta t$ can make sure that the output error is kept within a permitted range). In the steady state, both outputs are equal.

(2) The coefficients $\alpha_{1}(k), \alpha_{2}(k), \beta_{0}(k)$, and $\beta_{1}(k)$ are slowly time-varying.

(3) The range of these coefficients can be determined beforehand.

If the system is a minimum phase system, $\beta_{1}(k) u(k-1)$ in (7) can be left out.

According to Lemma 7, the characteristic model only can be applied to the system which is continuous differentiable to all the variables and where all partial derivatives are bounded. But in the system dynamic equations (2.1), the dead-zone function of the backlash model and the sign function $\operatorname{sgn}(\cdot)$ in the friction model are both indifferentiable. Thus, to establish the characteristic model of the system, the backlash model and the friction model should be smoothed.

According to the modeling method of backlash (see [29]), a continuous approximate dead-zone function is introduced as

$$
f^{*}(z)=z-a \alpha\left(\frac{2}{1+e^{-r z}}-1\right)
$$

where $a>0$ and $r>0$ are undetermined parameters in the backlash model. For analysing the degree of the approximation of the function (8) to (2), $\Delta f(z)$ is defined to be the difference between the two functions:

$$
\Delta f(z)= \begin{cases}-a \alpha\left(\frac{2}{1+e^{-r z}}-1\right)-\alpha, & z<-\alpha, \\ z-a \alpha\left(\frac{2}{1+e^{-r z}}-1\right), & |z| \leq \alpha, \\ -a \alpha\left(\frac{2}{1+e^{-r z}}-1\right)+\alpha, & z>\alpha .\end{cases}
$$




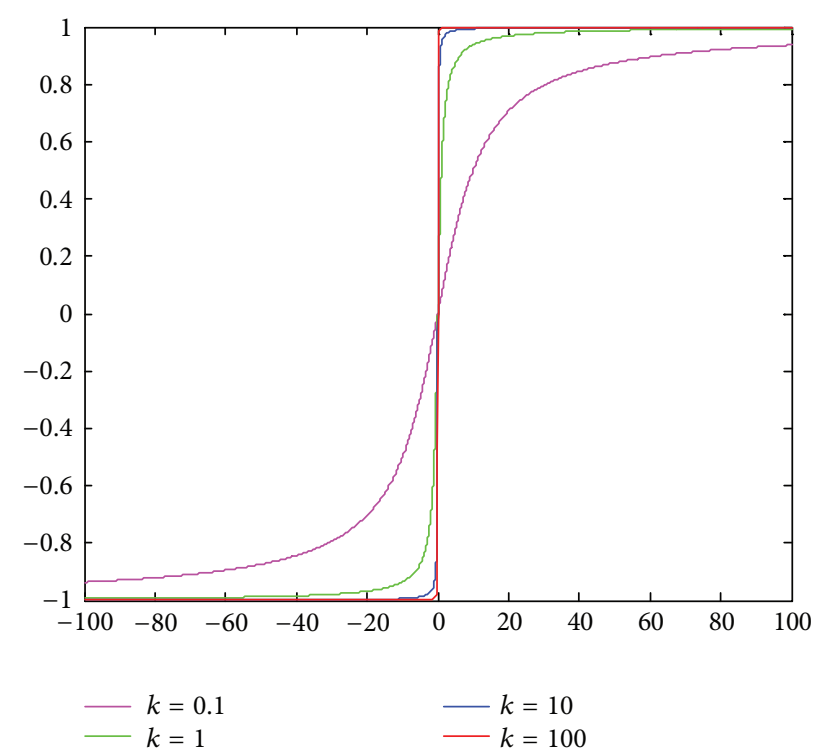

Figure 3: $(2 / \pi) \arctan (k v)$.

Lemma 8 (see [29]). When $a=1$ and $r=2 / \alpha$, the following conclusions can be obtained:

(1) $\lim _{z \rightarrow \infty} \Delta f(z)=0$;

(2) $f^{*}(z)$ is a monotonous increasing function;

(3) the area enclosed by $f^{*}(z)$ and $f(z)$ is the minimum;

(4) $|\Delta f(z)| \leq 2 \alpha e^{-r \alpha} /\left(1+e^{-r \alpha}\right)$.

Thus, the approximate dead-zone function $f^{*}(z)$ can be used to replace the dead-zone function in the system dynamic equations (2.1), and $\tau_{1}$ can be rewritten as

$$
\tau_{1}=k_{b}\left[z-\alpha\left(\frac{2}{1+e^{-r z}}-1\right)\right] .
$$

To make the friction model smooth, the function $(2 / \pi) \arctan (k v)$ is used to replace the sign function $\operatorname{sgn}(\cdot)$ in the friction model. $\Delta \mathrm{g} 2\left(k, \dot{\theta}_{m}\right)$ is defined as

$$
\Delta \mathrm{g}\left(k, \dot{\theta}_{m}\right)=\operatorname{sgn}\left(\dot{\theta}_{m}\right)-\frac{2}{\pi} \arctan \left(k \dot{\theta}_{m}\right)
$$

As shown in Figure 3, the function $\Delta \mathbf{g}\left(k, \dot{\theta}_{m}\right)$ is smaller and smaller with $k$ increasing.

Thus, the friction torque $\tau_{2}$ can be rewritten as

$$
\tau_{2}=\left[F_{c}+\left(F_{s}-F_{c}\right) e^{-\left(\dot{\theta}_{m} / \dot{\theta}_{s}\right)^{\delta}}\right] \frac{2}{\pi} \arctan \left(k \theta_{m}\right)+B \dot{\theta}_{m} .
$$

Define $x_{1}=\theta_{m}(t), x_{2}=\dot{\theta}_{m}(t), x_{3}=\theta_{d}(t), x_{4}=\dot{\theta}_{d}(t)$, and $x_{5}=I(t)$; then

$$
\begin{gathered}
\tau_{1}=k_{b}\left[\left(x_{1}-i x_{3}\right)-\alpha\left(\frac{2}{1+e^{-r\left(x_{1}-i x_{3}\right)}}-1\right)\right] \\
\tau_{2}=\left[F_{c}+\left(F_{s}-F_{c}\right) e^{-\left(x_{2} / \dot{\theta}_{s}\right)^{\delta}}\right] \frac{2}{\pi} \arctan \left(k x_{2}\right)+B x_{2} .
\end{gathered}
$$

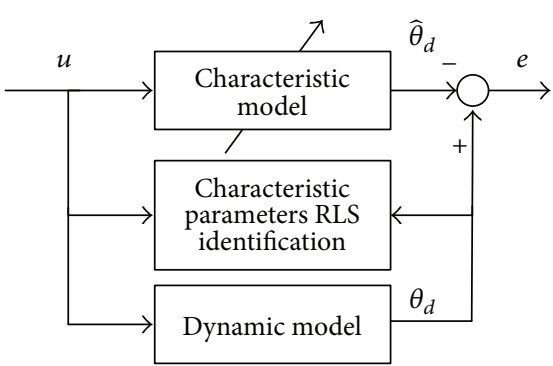

FIGURE 4: Scheme of verifying the characteristic model.

The system (2.1) can be expressed by state

$$
\begin{gathered}
\dot{x}_{1}=x_{2} \\
\dot{x}_{2}=\frac{1}{J_{m}}\left(k_{d} x_{5}-\tau_{1}-\tau_{2}-b_{m} x_{2}\right) \\
\dot{x}_{3}=x_{4} \\
\dot{x}_{4}=\frac{1}{J_{d}}\left(i \tau_{1}-b_{d} x_{4}\right) \\
\dot{x}_{5}=\frac{1}{L}\left(U-k_{e} x_{2}-R x_{5}\right) \\
y=x_{3} .
\end{gathered}
$$

The characteristic model of the system can be obtained based on Lemma 7 as

$$
\begin{aligned}
y(k)= & \alpha_{1}(k) y(k-1)+\alpha_{2}(k) y(k-2) \\
& +\beta_{0}(k) u(k-1)=\phi^{T}(k-1) \theta(k),
\end{aligned}
$$

where

$$
\begin{gathered}
\phi^{T}(k-1)=\left[\begin{array}{lll}
y(k-1) & y(k-2) & u(k-1)
\end{array}\right] \\
\theta^{T}(k)=\left[\begin{array}{lll}
\alpha_{1}(k) & \alpha_{2}(k) & \beta_{0}(k)
\end{array}\right]
\end{gathered}
$$

where the parameters $\alpha_{1}(k), \alpha_{2}(k)$, and $\beta_{0}(k)$ are all bounded and the bound can be determined beforehand according to Lemma 7. These coefficients are time-varying and need to be estimated online, so the recursive least squares (RLS) method with forgetting factor $f$ is applied. The recursive formula is shown as

$$
\begin{aligned}
K(k) & =\frac{P(k-1) \phi(k-1)}{f+\phi^{T}(k-1) P(k-1) \phi(k-1)} \\
\hat{\theta}(k) & =\hat{\theta}(k-1)+K(k)\left[y(k)-\phi^{T}(k-1) \hat{\theta}(k-1)\right] \\
P(k) & =\frac{1}{f}\left[I-K(k) \phi^{T}(k)\right] P(k-1) .
\end{aligned}
$$

To verify the effectiveness of the proposed characteristic model, the simulations of verifying the characteristic model are performed based on MATLAB. The output of the dynamic model is as the standard output. The scheme of verifying the characteristic model is shown in Figure 4. 


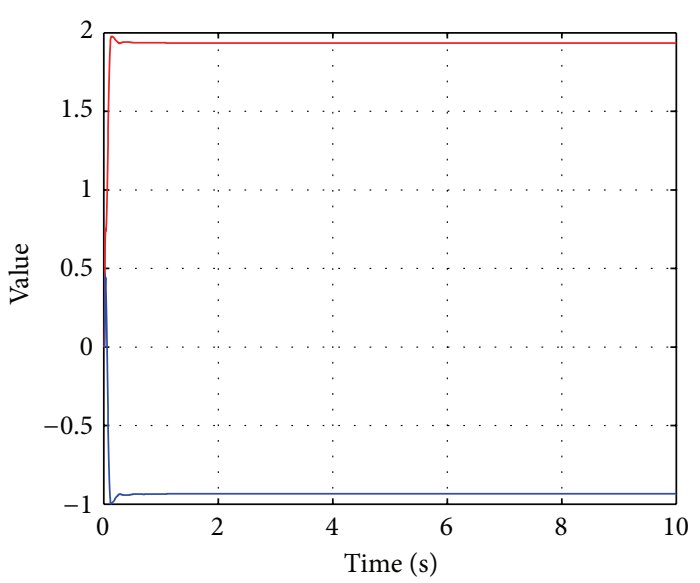

$-\widehat{\alpha}_{1}(k)$

$-\widehat{\alpha}_{2}(k)$

(a) $\widehat{\alpha}_{1}(k), \widehat{\alpha}_{2}(k)$

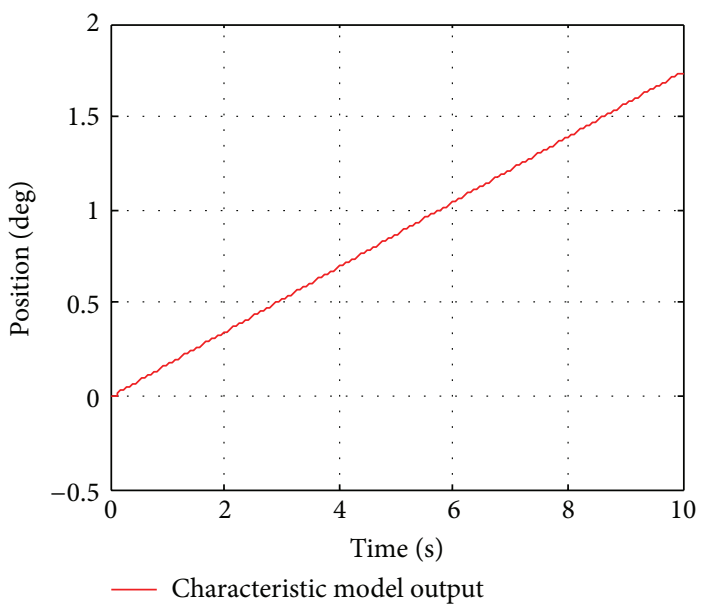

(c) Characteristic model output

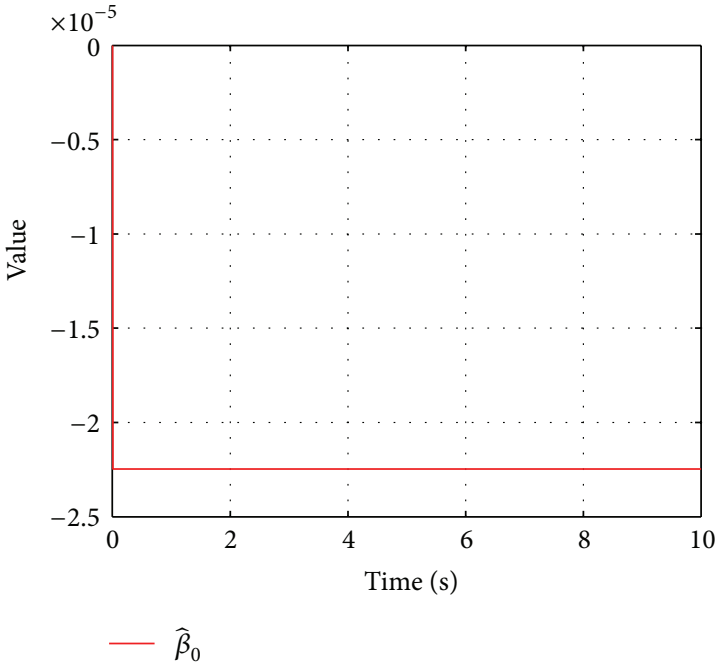

(b) $\widehat{\beta}_{0}(k)$

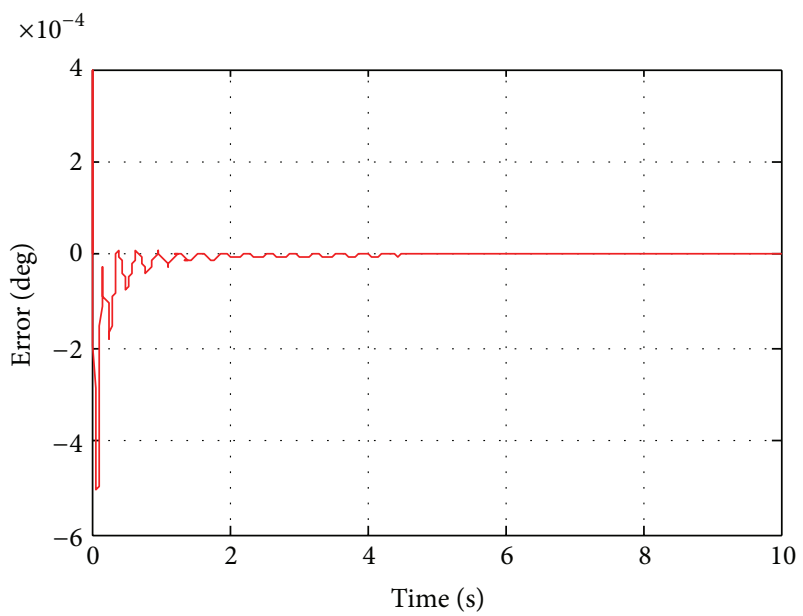

(d) $e(k)$

FIGURE 5: Verification results when $u(t)=10 \mathrm{~V}$.

The system parameters are as $L=0.0375 \mathrm{H}, R=$ $1.3 \Omega, k_{e}=67.2 \mathrm{~V} / \mathrm{krpm}, k_{d}=1.11 \mathrm{~N} \cdot \mathrm{m} / \mathrm{A}, k_{b}=7 \times$ $10^{4} \mathrm{~N} \cdot \mathrm{m} / \mathrm{rad}, J_{m}=0.00259 \mathrm{~kg} \cdot \mathrm{m}^{2}, J_{d}=23.12 \mathrm{~kg} \cdot \mathrm{m}^{2}, b_{m}=$ $0.015 \mathrm{~N} \cdot \mathrm{m} / \mathrm{krpm}, b_{d}=0.024 \mathrm{~N} \cdot \mathrm{m} / \mathrm{krpm}, i=238, \alpha=0.18^{\circ}$, and $r=1.7 / \alpha, k=10$.

The sampling period $\Delta t$ is $5 \mathrm{~ms}$. Define the error $e(k)$ between the output of the characteristic model $\theta_{d}$ and the standard output $\widehat{\theta}_{d}$ as

$$
e(k)=\theta_{d}(k)-\widehat{\theta}_{d}(k)=\theta_{d}(k)-\phi^{T}(k-1) \hat{\theta}(k) .
$$

The RLS method expressed in (17) is used to estimate the characteristic parameters; the initial values are selected as $\widehat{\boldsymbol{\theta}}(0)=10^{-3} \times\left[\begin{array}{lll}1 & 1 & 1\end{array}\right]^{T}, \mathbf{P}(0)=10^{6} \times \mathbf{I}_{3 \times 3}$, and the forgetting factor $f$ is 0.99 .

The results of verifying the characteristic model are shown in Figures 5-7.

(1) When $u(t)=10 \mathrm{~V}$, the results are shown in Figure 5 .
(2) When $u(t)=10 t \mathrm{~V}$, the results are shown in Figure 6 .

(3) When $u(t)=30 \sin (t) \mathrm{V}$, the results are shown in Figure 7.

According to Figures 5-7, the error between the characteristic model and the dynamic model is very small. The results indicate that the characteristic model can well describe the electromechanical system.

\section{Controller Design and Stability Analysis}

According to [25], the system error characteristic equation can be expressed as a second-order slowly time-varying difference equation:

$$
y_{e}(k+1)=\alpha_{1}(k) y_{e}(k)+\alpha_{2}(k) y_{e}(k-1)+\beta_{0}(k) u(k),
$$




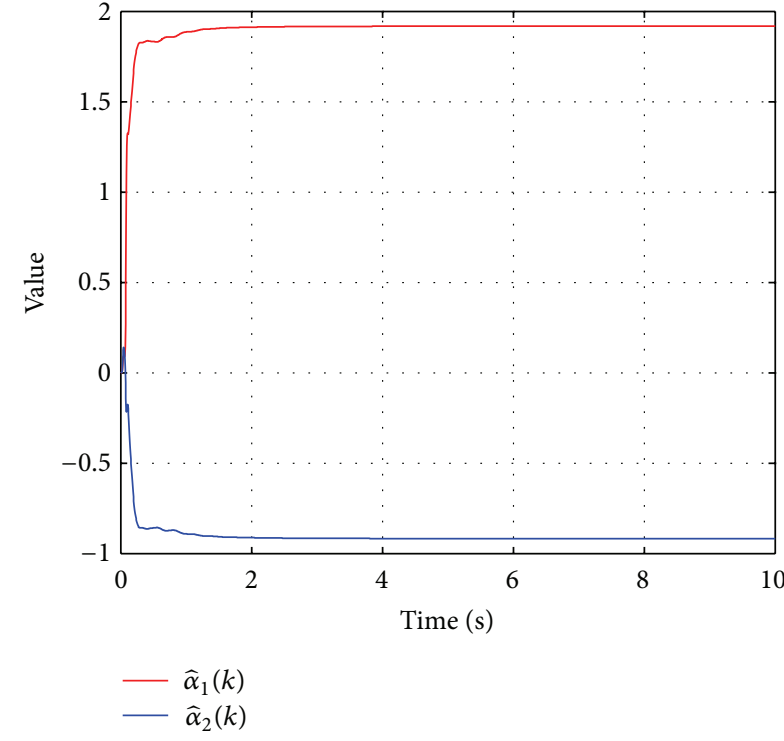

(a) $\widehat{\alpha}_{1}(k), \widehat{\alpha}_{2}(k)$

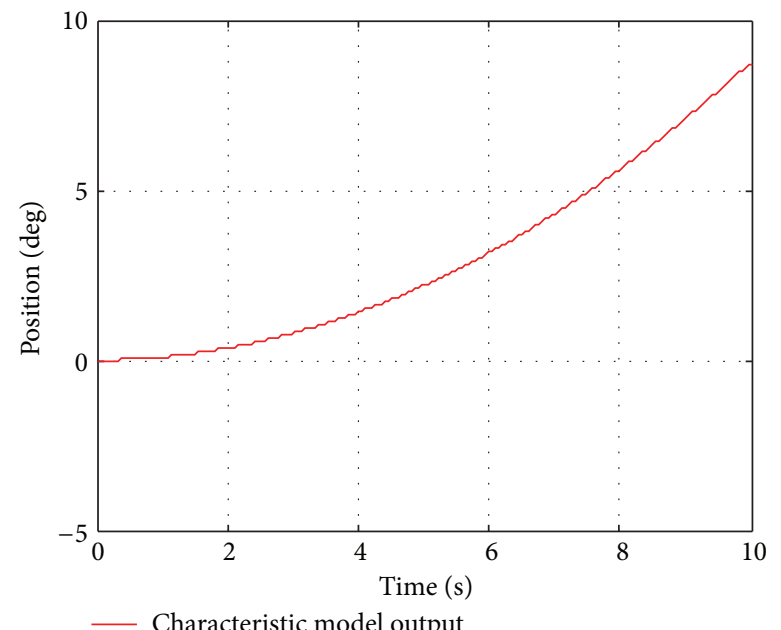

(c) Characteristic model output

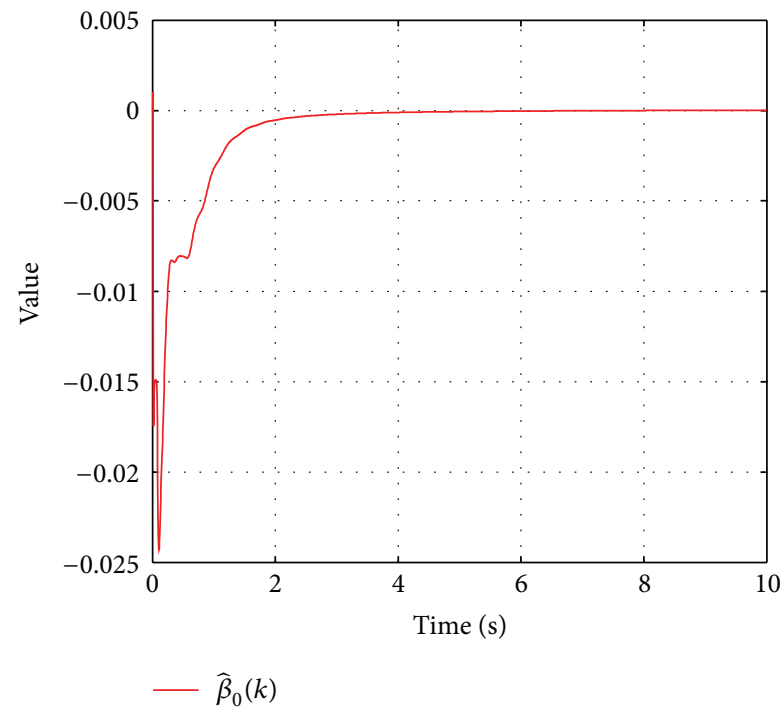

(b) $\widehat{\beta}_{0}(k)$

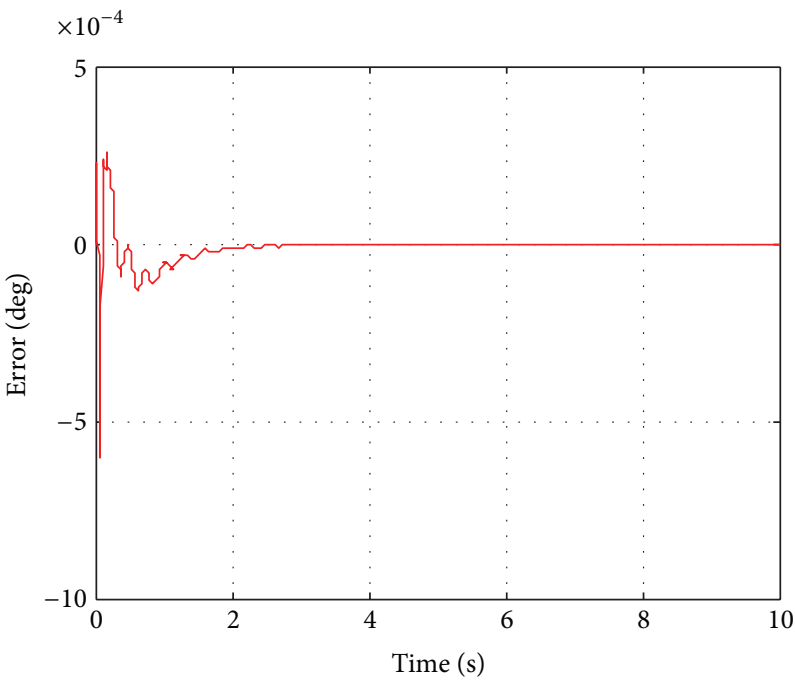

(d) $e(k)$

Figure 6: Verification results when $u(t)=10 t \mathrm{~V}$.

where $y_{e}(k)\left(y_{e}(k)=y(k)-y_{r}(k)\right)$ is the error output, $y(k)$ is the system input, and $u(k)$ is the controller input which is designed as

$$
u(k)=u_{1}(k)+u_{2}(k),
$$

where $u_{1}(k)$ and $u_{2}(k)$ are the golden-section adaptive control law, and the integral control law, respectively, which satisfy

$$
\begin{gathered}
u_{1}(k)=-\frac{\left[l_{1} \widehat{\alpha}_{1}(k) y_{e}(k)+l_{2} \widehat{\alpha}_{2}(k) y_{e}(k-1)\right]}{\widehat{\beta}_{0}(k)}, \\
u_{2}(k)=u_{2}(k-1)+\alpha_{0}(k) y_{e}(k) \\
=u_{2}(k-2)+\alpha_{0}(k-1) y_{e}(k-1)+\alpha_{0}(k) y_{e}(k),
\end{gathered}
$$

where $l_{1}=0.382$ and $l_{2}=0.618$ are the golden-section feedback coefficients, $\widehat{\alpha}_{1}(k), \widehat{\alpha}_{2}(k)$, and $\widehat{\beta}_{0}(k)$ are the estimates of $\alpha_{1}(k), \alpha_{2}(k)$, and $\beta_{0}(k)$, and $-1<\alpha_{0}(k)<0$ is a variable integral coefficient. Take (20) and (21) into (19) to obtain

$$
\begin{aligned}
y_{e}(k+1)= & -f_{1}(k) y_{e}(k)-f_{2}(k) y_{e}(k-1) \\
& -f_{3}(k) u_{2}(k-2),
\end{aligned}
$$

where

$$
f_{1}(k)=l_{1} \frac{\beta_{0}(k) \widehat{\alpha}_{1}(k)}{\widehat{\beta}_{0}(k)}-\alpha_{1}(k)-\alpha_{0}(k) \beta_{0}(k),
$$




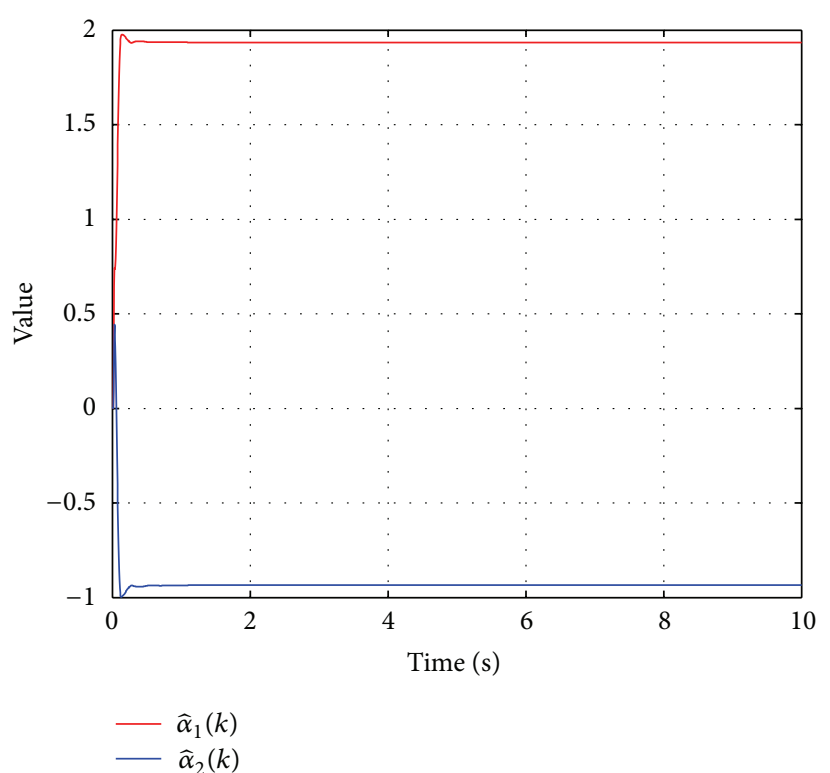

(a) $\widehat{\alpha}_{1}(k), \quad \widehat{\alpha}_{2}(k)$

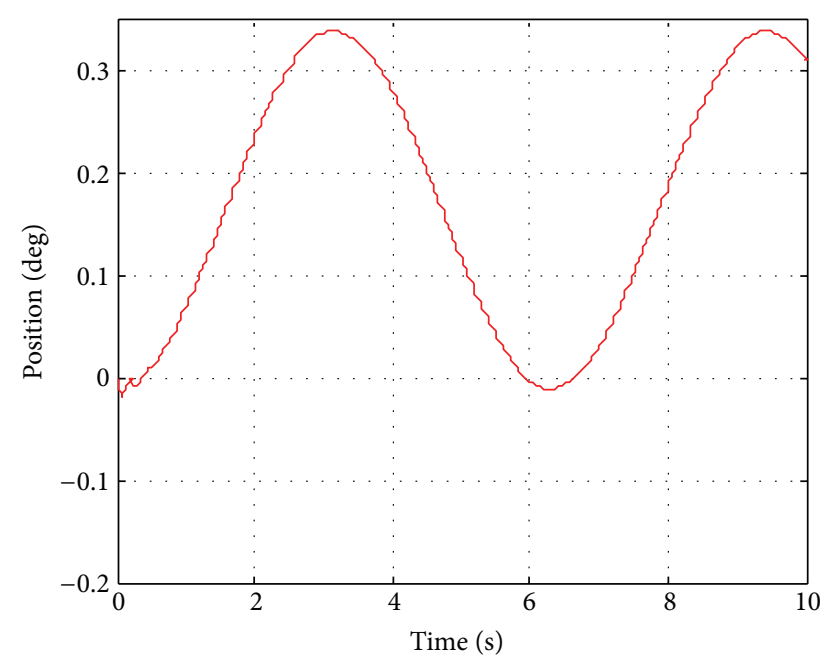

- Characteristic model output

(c) Characteristic model output

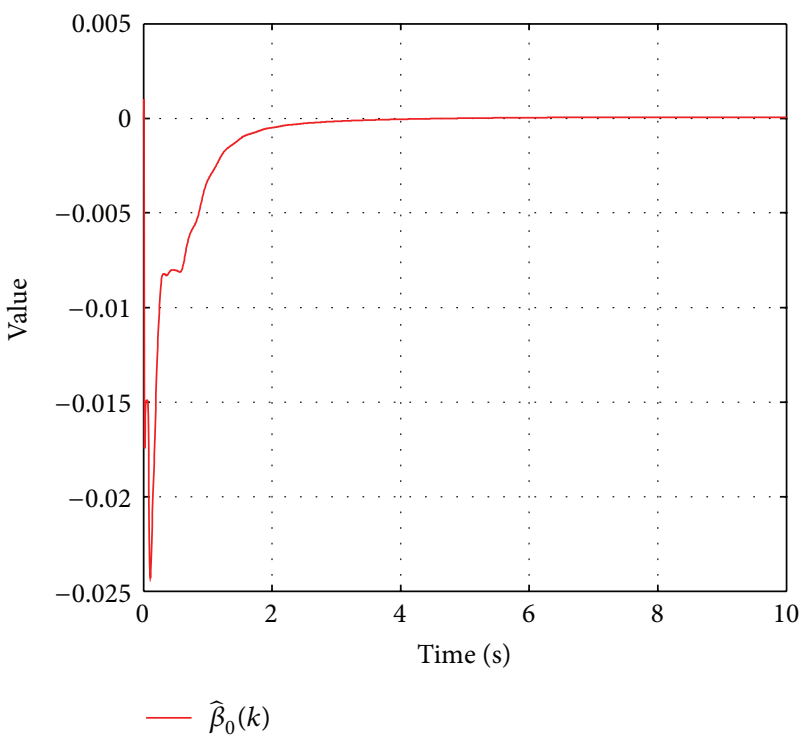

(b) $\widehat{\beta}_{0}(k)$

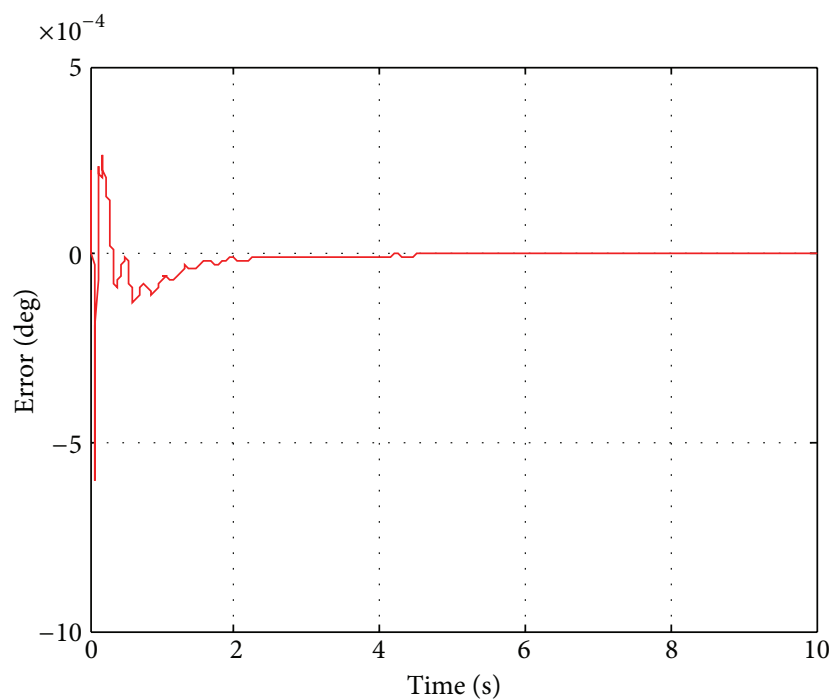

(d) $e(k)$

FIgURE 7: Verification results when $u(t)=30 \sin (t)$ V.

$$
\begin{gathered}
f_{2}(k)=l_{2} \frac{\beta_{0}(k) \widehat{\alpha}_{2}(k)}{\widehat{\beta}_{0}(k)}-\alpha_{2}(k)-\alpha_{0}(k-1) \beta_{0}(k), \\
f_{3}(k)=-\beta_{0}(k) .
\end{gathered}
$$

Define $Y(k)=\left[\begin{array}{lll}y_{0}(k-1) & y_{e}(k) & y_{e}(k+1)\end{array}\right]^{T}$, where $y_{0}(k-1)=u_{2}(k-1)$ so that the system (22) can be expressed as

$$
Y(k+1)=A(k+1) Y(k),
$$

where

$$
A(k+1)=\left[\begin{array}{ccc}
1 & \alpha_{0} & 0 \\
0 & 0 & 1 \\
-f_{3}(k+1) & -f_{2}(k+1) & -f_{1}(k+1)
\end{array}\right] .
$$

In order to analyze the uniform asymptotic stability of system (22), the following three lemmas are introduced.

Lemma 9 (see [30]). Assume that a linear time-varying discrete system can be written as

$$
Y(k+1)=A(k+1) Y(k),
$$


where $A(k+1) \in R^{n \times n}, Y(k) \in R^{n \times 1}$, and the origin is the equilibrium state of the system. For a given uniformly bounded, positive definite symmetric matrix $P(k)$, if $Q(k)$ which can be obtained by

$$
A^{T}(k+1) P(k+1) A(k+1)-P(k)=-Q(k)
$$

is a uniformly bounded, positive definite symmetric matrix, then the equilibrium state of the system (26) is uniformly asymptotically stable according to Lyapunov theory.

Lemma 10 (see [30]). Assume that $B(k)=\left(b_{i j}(k)\right)$ is a symmetric matrix which satisfies

(1) every element and principal minor of $B(k)$ can be expressed as a continuous function about $f_{i}(k)$ or a constant;

(2) $f_{i}(k)(i=1,2, \ldots, N)$ belongs to a finite closed interval, where $N$ is a finite positive integer;

(3) all principal minors of $B(k)$ are greater than zero.

Then $B(k)$ is a uniformly bounded, positive definite symmetric matrix.

Lemma 11 (see [30]). Assume that $M(k) \in R$ and $0<M(k)<$ $M$, where $M$ is a positive constant. Assume that $B(k+1), C(k+$ $1), D(k), E_{0}(k), E_{1}(k)$, and $F(k)$ satisfy

$$
\begin{aligned}
E_{0}(k)= & M(k) B^{2}(k+1)+E_{1}(k) B(k+1) \\
& -M(k) C^{2}(k+1)+F(k) C(k+1)+D(k), \\
E_{1}(k)= & -2 M(k) B(k+1)-2 M(k) C(k+1)+F(k) .
\end{aligned}
$$

The quadratic trinomial about $Z(k)$ is

$$
-M(k) Z^{2}(k)+E_{1}(k) Z(k)+E_{0}(k)
$$

whose discriminant root is $\Delta(k)=E_{1}^{2}(k)+4 M(k) E_{0}(k)=$ $F^{2}(k)+4 M(k) D(k)$. When

$$
\Delta(k)>0
$$

$$
\frac{E_{1}(k)-\sqrt{\Delta(k)}}{2 M(k)}<Z(k)<\frac{E_{1}(k)+\sqrt{\Delta(k)}}{2 M(k)},
$$

the quadratic trinomial (30) is greater than zero.

Design the symmetric matrix $P(k)$ as

$$
p_{i i}(k)=p_{i i} \quad(i=1,2,3),
$$

where $p_{11}, p_{22}$, and $p_{33}$ are all positive constants and $0<p_{11}<$ $p_{22}<p_{33}:$

$$
\begin{aligned}
& p_{12}(k)=p_{21}(k)=\varepsilon_{3} f_{3}(k), \\
& p_{13}(k)=p_{31}(k)=\varepsilon_{2} f_{2}(k), \\
& p_{23}(k)=p_{32}(k)=\varepsilon_{1} f_{1}(k),
\end{aligned}
$$

where $\varepsilon_{1}, \varepsilon_{2}$, and $\varepsilon_{3}$ are positive constants and $0<\varepsilon_{i} \leq$ $\left(p_{11} / M_{i} \sqrt{5}\right)(i=1,2,3)$, where $M_{i}$ is the bound of $f_{i}(k)$; that is, $\left|f_{i}(k)\right|<M_{i}$.

Then the $Q(k)$ can be calculated as

$$
\begin{aligned}
\left(Q_{i j}(k)\right)_{3 \times 3} & \triangleq Q(k) \\
& =-A^{T}(k+1) P(k+1) A(k+1)+P(k),
\end{aligned}
$$

where

$$
\begin{aligned}
Q_{11}(k)= & 2 p_{13}(k+1) f_{3}(k+1)-p_{33} f_{3}^{2}(k+1), \\
Q_{12}(k)= & Q_{21}(k) \\
= & p_{12}(k)-\alpha_{0} p_{11}+\alpha_{0} p_{13}(k+1) f_{3}(k+1) \\
& +p_{13}(k+1) f_{2}(k+1)-p_{33} f_{2}(k+1) f_{3}(k+1), \\
Q_{13}(k)= & Q_{31}(k) \\
= & p_{13}(k)-p_{12}(k+1)+p_{23}(k+1) f_{3}(k+1) \\
& +p_{13}(k+1) f_{1}(k+1)-p_{33} f_{1}(k+1) f_{3}(k+1), \\
Q_{22}(k)= & p_{22}-\alpha_{0}^{2} p_{11}+2 \alpha_{0}^{2} p_{13}(k+1) f_{2}(k+1) \\
& -p_{33} f_{2}^{2}(k+1), \\
Q_{23}(k)= & Q_{32}(k) \\
= & p_{23}(k)-\alpha_{0} p_{12}(k+1)+\alpha_{0} p_{13}(k+1) f_{1}(k+1) \\
& +p_{23}(k+1) f_{2}(k+1)-p_{33} f_{1}(k+1) f_{2}(k+1), \\
Q_{33}(k)= & p_{33}-p_{22}+2 p_{23}(k+1) f_{1}(k+1) \\
& -p_{33} f_{1}^{2}(k+1) .
\end{aligned}
$$

Theorem 12. The sufficient conditions which guarantee the uniform asymptotic stability of the time-varying discrete system (15) are

(1) $f_{1}(k), f_{2}(k)$, and $f_{3}(k)$ satisfy

$$
\begin{gathered}
\left|f_{1}(k)\right|<\sqrt{\frac{p_{33}-p_{22}}{p_{33}}}=M_{1} \\
\left|f_{2}(k)\right|<\sqrt{\frac{p_{22}-\alpha_{0}^{2} p_{11}}{p_{33}}}, \quad f_{2}(k) \neq 0 \\
\frac{\varepsilon_{2}}{p_{33}}\left(f_{2}(k+1)-\left|f_{2}(k+1)\right|\right) \\
<f_{3}(k)<\frac{\varepsilon_{2}}{p_{33}}\left(f_{2}(k+1)+\left|f_{2}(k+1)\right|\right) .
\end{gathered}
$$


(2) The change rate of $f_{1}(k), f_{2}(k)$, and $f_{3}(k)$ satisfy

$$
\begin{aligned}
& \frac{\left(S_{23} / 2\right)-\sqrt{M_{\mathrm{Q} 2}(k)\left(Q_{11}(k) Q_{33}(k)-Q_{13}^{2}(k)\right)}}{Q_{11}(k)}<-\varepsilon_{1} \Delta f_{1} \\
& <\frac{\left(S_{23} / 2\right)+\sqrt{M_{\mathrm{Q} 2}(k)\left(Q_{11}(k) Q_{33}(k)-Q_{13}^{2}(k)\right)}}{Q_{11}(k)} \\
& \frac{S_{13}}{2}-\sqrt{Q_{11}(k) Q_{33}(k)} \\
& \quad<-\varepsilon_{2} \Delta f_{2}(k)<\frac{S_{13}}{2}+\sqrt{Q_{11}(k) Q_{33}(k)} \\
& \frac{S_{12}}{2}-\sqrt{Q_{11}(k) Q_{22}(k)} \\
& <-\varepsilon_{3} \Delta f_{3}(k)<\frac{S_{12}}{2}+\sqrt{Q_{11}(k) Q_{22}(k)},
\end{aligned}
$$

where

$$
\begin{gathered}
S_{23}=-2 Q_{11}(k) p_{23}(k+1)-2 Q_{11}(k) \\
\times\left(-\alpha_{0} p_{12}(k+1)+\alpha_{0} p_{13}(k+1) f_{1}(k+1)\right. \\
+p_{23}(k+1) f_{2}(k+1) \\
\left.\quad-p_{33} f_{1}(k+1) f_{2}(k+1)\right)+2 Q_{12}(k) Q_{13}(k), \\
S_{13}=-2 p_{13}(k+1) \\
-2\left(-p_{12}(k+1)+p_{23}(k+1) f_{3}(k+1)\right. \\
\quad+p_{13}(k+1) f_{1}(k+1) \\
\left.\quad-p_{33} f_{1}(k+1) f_{3}(k+1)\right), \\
S_{12}=-2 p_{12}(k+1) \\
-2\left(-\alpha_{0} p_{11}+\alpha_{0} p_{13}(k+1) f_{3}(k+1)\right. \\
+p_{13}(k+1) f_{2}(k+1) \\
\left.\quad-p_{33} f_{2}(k+1) f_{3}(k+1)\right) \\
M_{\mathrm{Q} 2}(k)=Q_{11}(k) Q_{22}(k)-Q_{12}^{2}(k) \\
\Delta f_{i}(k)=f_{i}(k+1)-f_{i}(k) \quad(i=1,2,3) .
\end{gathered}
$$

Remark 13. Due to $-1<\alpha_{0}(k)<0$, we obtain $\left|f_{2}(k)\right|<$ $\sqrt{\left(p_{22}-\alpha_{0}^{2} p_{11}\right) / p_{33}}<\sqrt{p_{22} / p_{33}}=M_{2}$ by (37). According to (38), we get

$$
\begin{array}{ll}
0<f_{3}(k)<\frac{2 \varepsilon_{2}}{p_{33}} f_{2}(k+1) \quad f_{2}(k+1)>0 \\
\frac{2 \varepsilon_{2}}{p_{33}} f_{2}(k+1)<f_{3}(k)<0 \quad f_{2}(k+1)<0 .
\end{array}
$$

So $\left|f_{3}(k)\right|<\left(2 \varepsilon_{2} / p_{33}\right)\left|f_{2}(k+1)\right|<\left(2 / p_{33}\right)\left(p_{11} / M_{2} \sqrt{5}\right) M_{2}=$ $2 \sqrt{5} p_{11} / 5 p_{33}=M_{3}$.
Remark 14. The bound of $f_{2}(k)$ and $f_{3}(k)$ is related to $\alpha_{0}(k)$ and $f_{2}(k+1)$, respectively, in (37) and (38). In order to make the conditions (37) and (38) possible to be satisfied in the operation of the system, we reduce the range for

$$
\begin{gathered}
\delta<\left|f_{2}(k)\right|<\sqrt{\frac{p_{22}-p_{11}}{p_{33}}} \\
0<f_{3}(k)<\frac{2 \varepsilon_{2}}{p_{33}} \delta \quad f_{2}(k+1)>0 \\
-\frac{2 \varepsilon_{2}}{p_{33}} \delta<f_{3}(k)<0 \quad f_{2}(k+1)<0,
\end{gathered}
$$

where $\delta$ is a positive constant. In this way, the upper bound and lower bound of $f_{2}(k)$ and $f_{3}(k)$ are constant and they are possible to implement in real system.

Proof of Theorem 12. The first-order principal minor of $P(k)$ satisfies

$$
M_{p 1}(k)=p_{11}, \quad p_{11}>0 .
$$

The second-order principal minor of $P(k)$ satisfies

$$
\begin{aligned}
M_{p 2}(k) & =p_{11} p_{22}-p_{12}^{2}(k)=p_{11} p_{22}-\varepsilon_{3}^{2} f_{3}^{2}(k) \\
& >p_{11} p_{22}-\frac{p_{11}^{2}}{5 M_{3}^{2}} M_{3}^{2}>p_{11}^{2}-\frac{p_{11}^{2}}{5}>0 .
\end{aligned}
$$

The third-order principal minor of $P(k)$ satisfies

$$
\begin{aligned}
M_{p 3}(k)= & p_{11} p_{22} p_{33}+2 \varepsilon_{1} \varepsilon_{2} \varepsilon_{3} f_{1}(k) f_{2}(k) f_{3}(k) \\
& -p_{11} \varepsilon_{1}^{2} f_{1}^{2}(k)-p_{22} \varepsilon_{2}^{2} f_{2}^{2}(k)-p_{33} \varepsilon_{3}^{2} f_{3}^{2}(k) \\
> & p_{11} p_{22} p_{33}-2 \frac{p_{11}}{M_{1} \sqrt{5}} \frac{p_{11}}{M_{2} \sqrt{5}} \frac{p_{11}}{M_{3} \sqrt{5}} M_{1} M_{2} M_{3} \\
& -p_{11} \frac{p_{11}^{2}}{5 M_{1}^{2}} M_{1}^{2}-p_{22} \frac{p_{11}^{2}}{5 M_{2}^{2}} M_{2}^{2}-p_{33} \frac{p_{11}^{2}}{5 M_{3}^{2}} M_{3}^{2} \\
> & \frac{2}{5} p_{11}^{3}+\frac{1}{5} p_{11}^{3}+\frac{1}{5} p_{22} p_{11}^{2}+\frac{1}{5} p_{33} p_{11}^{2}-\frac{2}{5 \sqrt{5}} p_{11}^{3} \\
& -\frac{1}{5} p_{11}^{3}-\frac{1}{5} p_{22} p_{11}^{2}-\frac{1}{5} p_{33} p_{11}^{2}>0 .
\end{aligned}
$$

As a result, $P(k)$ is a uniformly bounded, positive definite symmetric matrix by Lemma 10 .

The first-order principal minor of $Q(k)$ is

$$
\begin{aligned}
M_{\mathrm{Q} 1}(k)= & Q_{11}(k)=2 \varepsilon_{2} f_{2}(k+1) f_{3}(k+1)-p_{33} f_{3}^{2}(k+1) \\
= & -p_{33}\left[f_{3}(k+1)-\frac{\varepsilon_{2} f_{2}(k+1)+\varepsilon_{2}\left|f_{2}(k+1)\right|}{p_{33}}\right] \\
& \times\left[f_{3}(k+1)-\frac{\varepsilon_{2} f_{2}(k+1)-\varepsilon_{2}\left|f_{2}(k+1)\right|}{p_{33}}\right] .
\end{aligned}
$$


According to (38) and $f_{2}(k) \neq 0$ in (37), we can obtain $M_{\mathrm{Q} 1}(k)=Q_{11}(k)>0$.

\section{Define}

$$
\begin{aligned}
a_{12}(k+1)= & Q_{12}(k)-p_{12}(k) \\
= & -\alpha_{0} p_{11}+\alpha_{0} p_{13}(k+1) f_{3}(k+1) \\
& +p_{13}(k+1) f_{2}(k+1) \\
& -p_{33} f_{2}(k+1) f_{3}(k+1) .
\end{aligned}
$$

According to (43), the second-order principal minor of $Q(k)$ is

$$
\begin{aligned}
M_{\mathrm{Q} 2}(k)= & -\left[p_{12}(k)+a_{12}(k+1)\right]^{2}+Q_{11}(k) Q_{22}(k) \\
= & -\left[p_{12}(k)-p_{12}(k+1)\right]^{2} \\
& +S_{12}\left[p_{12}(k)-p_{12}(k+1)\right]+T_{12},
\end{aligned}
$$

where

$$
\begin{aligned}
S_{12}= & -2 p_{12}(k+1)-2 a_{12}(k+1), \\
T_{12}= & p_{12}^{2}(k+1)+S_{12} p_{12}(k+1) \\
& -a_{12}^{2}(k+1)+Q_{11}(k) Q_{22}(k) .
\end{aligned}
$$

$S_{12}$ and $T_{12}$ can be expressed in the form of (29) and (28):

$$
\begin{array}{cc}
B(k+1)=p_{12}(k+1), & C(k+1)=a_{12}(k+1), \\
D(k)=Q_{11}(k) Q_{22}(k), & F(k)=0,
\end{array}
$$

Then the discriminant root of the quadratic trinomial (52) about $\left[p_{12}(k)-p_{12}(k+1)\right]$ is

$$
\begin{aligned}
\Delta_{12}(k) & =F^{2}(k)+4 M(k) D(k)=4 Q_{11}(k) Q_{22}(k) \\
& =4 M_{Q 1}(k) Q_{22}(k),
\end{aligned}
$$

where

$$
Q_{22}(k)=p_{22}-\alpha_{0}^{2} p_{11}+2 \alpha_{0}^{2} \varepsilon_{2} f_{2}^{2}(k+1)-p_{33} f_{2}^{2}(k+1) .
$$

We can get $Q_{22}(k)>0$ by (37), so $\Delta_{12}(k)>0$. Then we have $M_{\mathrm{Q} 2}(k)>0$ according to (41) and Lemma 11 .

The third-order principal minor of $P(k)$ is

$$
\begin{aligned}
M_{\mathrm{Q} 3}(k)= & -Q_{11}(k) Q_{23}^{2}(k)+2 Q_{12}(k) Q_{13}(k) Q_{23}(k) \\
& -Q_{22}(k) Q_{13}^{2}(k)+Q_{33}(k) M_{Q 2}(k) .
\end{aligned}
$$

Define

$$
\begin{aligned}
a_{23}(k+1)= & Q_{23}(k)-p_{23}(k) \\
= & -\alpha_{0} p_{12}(k+1)+\alpha_{0} p_{13}(k+1) f_{1}(k+1) \\
& +p_{23}(k+1) f_{2}(k+1) \\
& -p_{33} f_{1}(k+1) f_{2}(k+1) .
\end{aligned}
$$

We get

$$
\begin{aligned}
M_{\mathrm{Q} 3}(k)= & -Q_{11}(k)\left[p_{23}(k)+a_{23}(k+1)\right]^{2} \\
& +2 Q_{12}(k) Q_{13}(k) a_{23}(k+1)-Q_{22}(k) Q_{13}^{2}(k) \\
& +Q_{33}(k) M_{Q 2}(k) \\
= & -Q_{11}(k)\left[p_{23}(k)-p_{23}(k+1)\right]^{2} \\
& +S_{23}\left[p_{23}(k)-p_{23}(k+1)\right]+T_{23}
\end{aligned}
$$

where

$$
\begin{aligned}
S_{23}= & -2 Q_{11}(k) p_{23}(k+1)-2 Q_{11}(k) a_{23}(k+1) \\
& +2 Q_{12}(k) Q_{13}(k), \\
T_{23}= & Q_{11}(k) p_{23}^{2}(k+1)+S_{23} p_{23}(k+1) \\
& -Q_{11}(k) a_{23}^{2}(k+1)+2 Q_{12}(k) Q_{13}(k) a_{23}(k+1) \\
& -Q_{22}(k) Q_{13}^{2}(k)+Q_{33}(k) M_{Q 2}(k) .
\end{aligned}
$$

$S_{23}$ and $T_{23}$ can be expressed in the form of (29) and (28):

$$
\begin{gathered}
M(k)=Q_{11}(k), \quad B(k+1)=p_{23}(k+1), \\
C(k+1)=a_{23}(k+1), \\
D(k)=-Q_{22}(k) Q_{13}^{2}(k)+Q_{33}(k) M_{\mathrm{Q} 2}(k), \\
F(k)=2 Q_{12}(k) Q_{13}(k) .
\end{gathered}
$$

Then the discriminant root of the quadratic trinomial (59) about $\left[p_{23}(k)-p_{23}(k+1)\right]$ is

$$
\begin{aligned}
\Delta_{23}(k)= & F^{2}(k)+4 M(k) D(k)=4 Q_{12}^{2}(k) Q_{13}^{2}(k) \\
& +4 Q_{11}(k)\left[-Q_{22}(k) Q_{13}^{2}(k)+Q_{33}(k) M_{\mathrm{Q} 2}(k)\right] \\
= & 4 Q_{13}^{2}(k)\left[Q_{12}^{2}(k)-Q_{11}(k) Q_{22}(k)\right] \\
& +4 Q_{11}(k) Q_{33}(k) M_{Q_{2}}(k) \\
= & 4 M_{Q_{2}}(k)\left[Q_{11}(k) Q_{33}(k)-Q_{13}^{2}(k)\right] .
\end{aligned}
$$

Define

$$
\begin{aligned}
a_{13}(k+1)= & Q_{13}(k)-p_{13}(k) \\
= & -p_{12}(k+1)+p_{23}(k+1) f_{3}(k+1) \\
& +p_{13}(k+1) f_{1}(k+1) \\
& -p_{33} f_{1}(k+1) f_{3}(k+1) .
\end{aligned}
$$




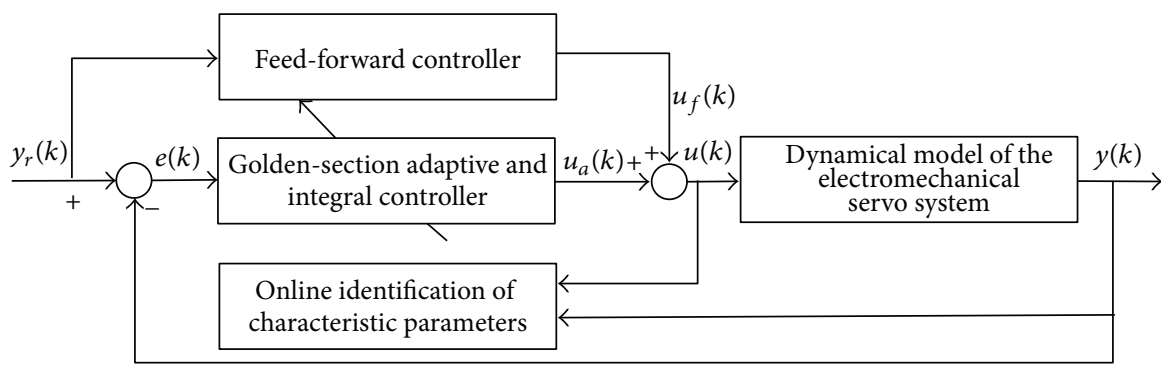

Figure 8: Structure of the closed-loop system.

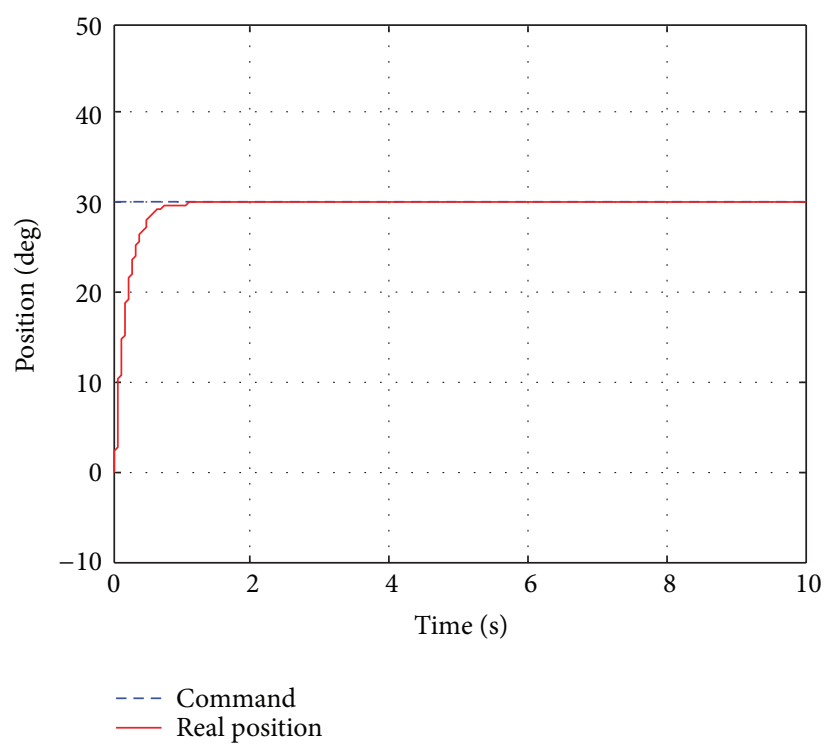

(a) Position tracking response

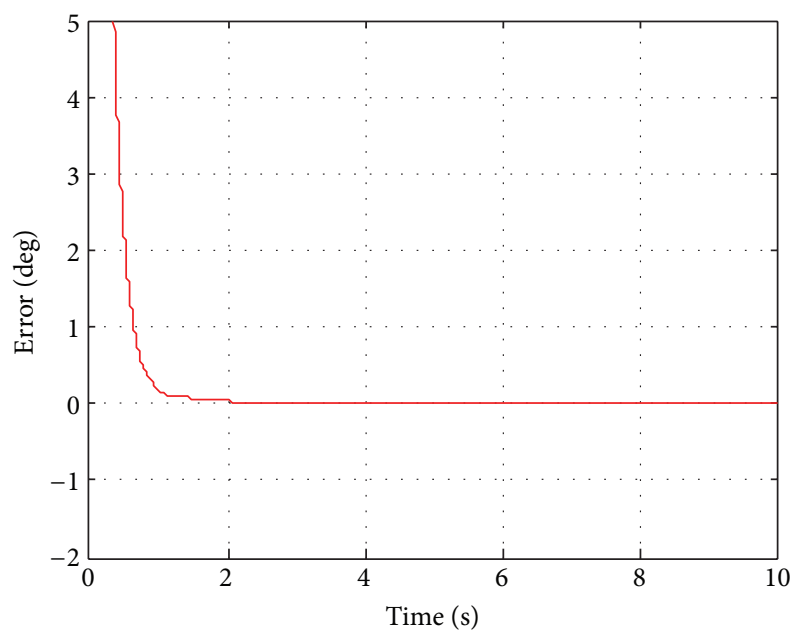

(b) Tracking error

Figure 9: Simulation results for the step signal input.

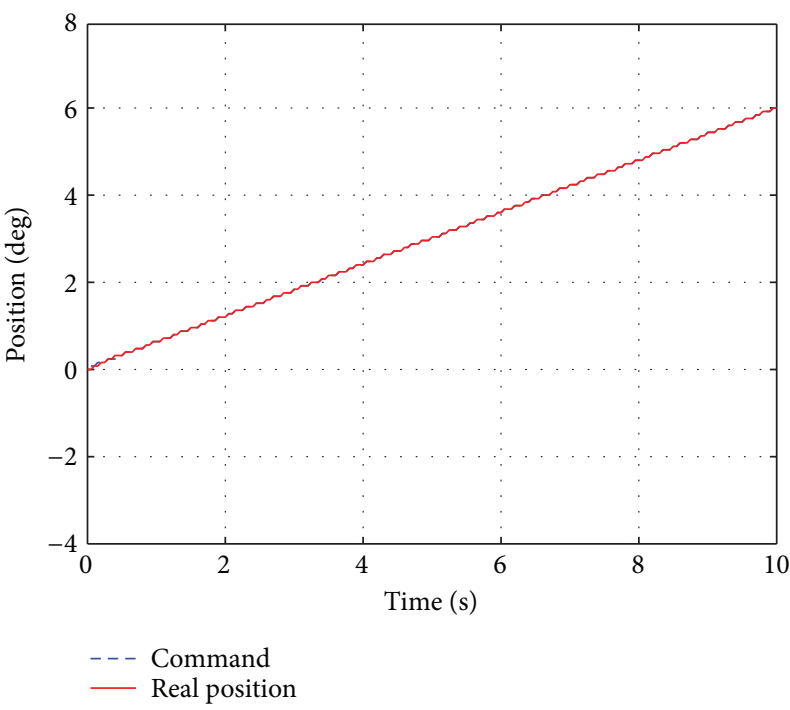

(a) Position tracking response

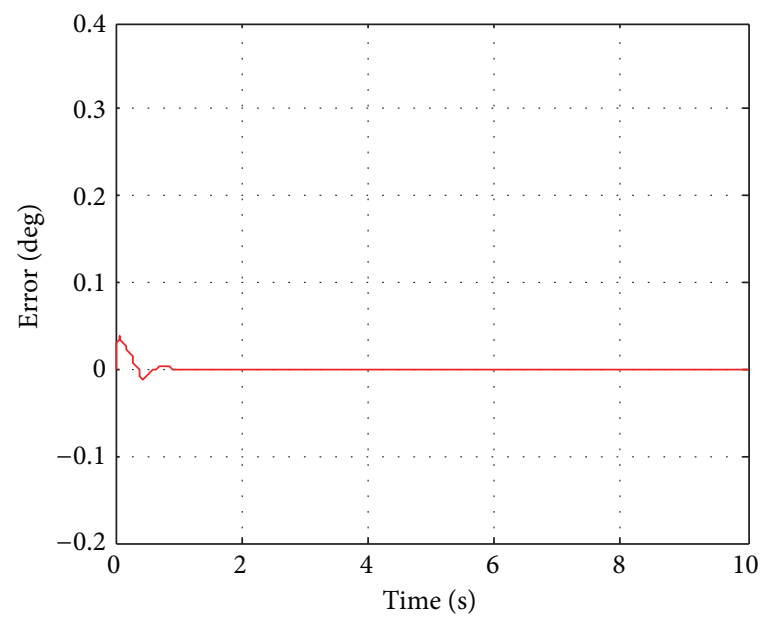

(b) Tracking error

FIGURE 10: Simulation results for the $0.6^{\circ} / \mathrm{s}$ slope signal input. 


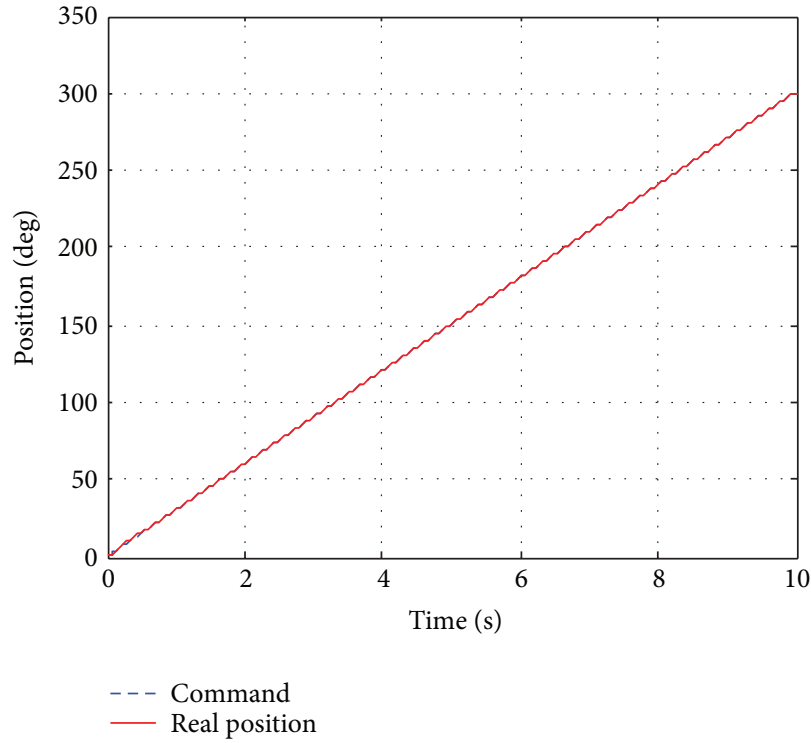

(a) Position tracking response

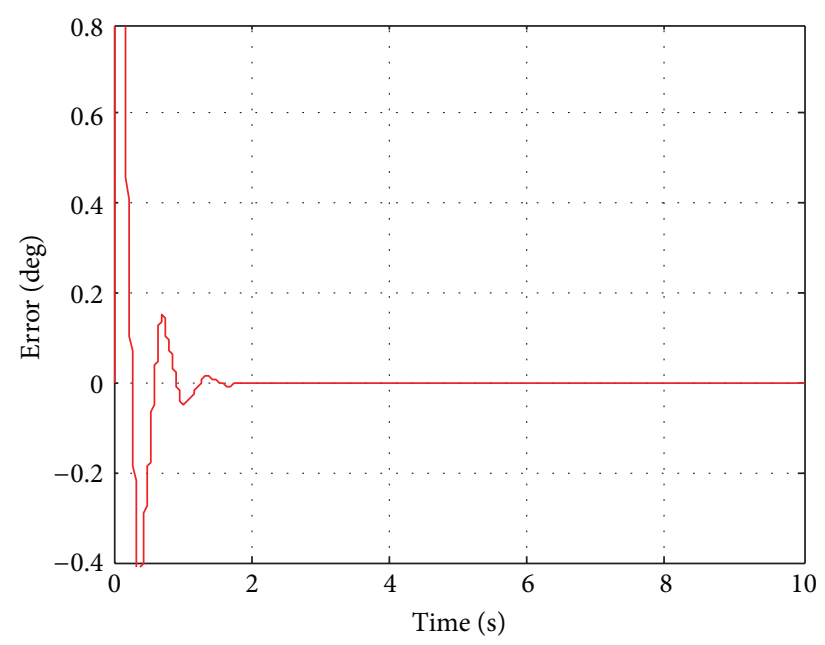

(b) Tracking error

FIGURE 11: Simulation results for the $30^{\circ} / \mathrm{s}$ slope signal input.

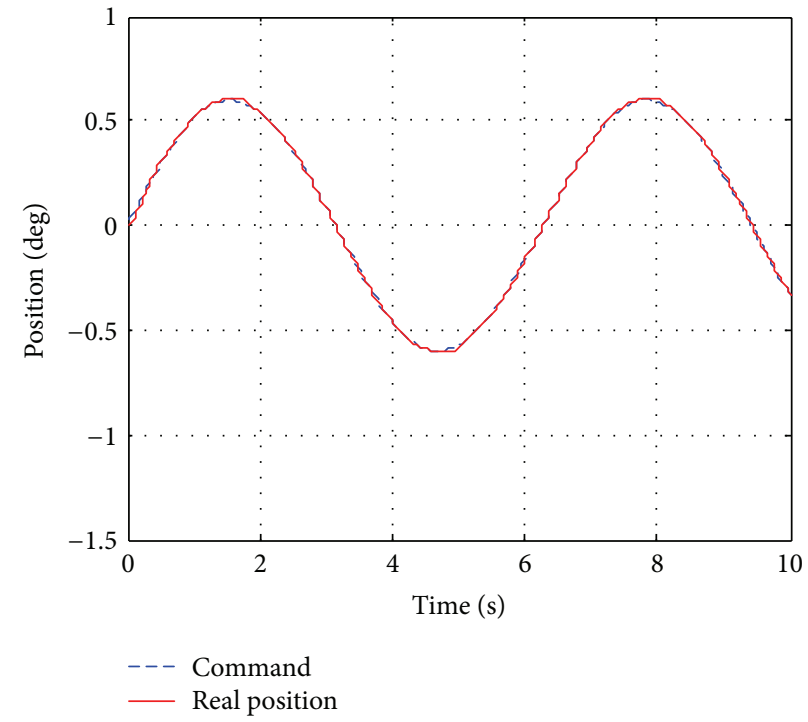

(a) Position tracking response

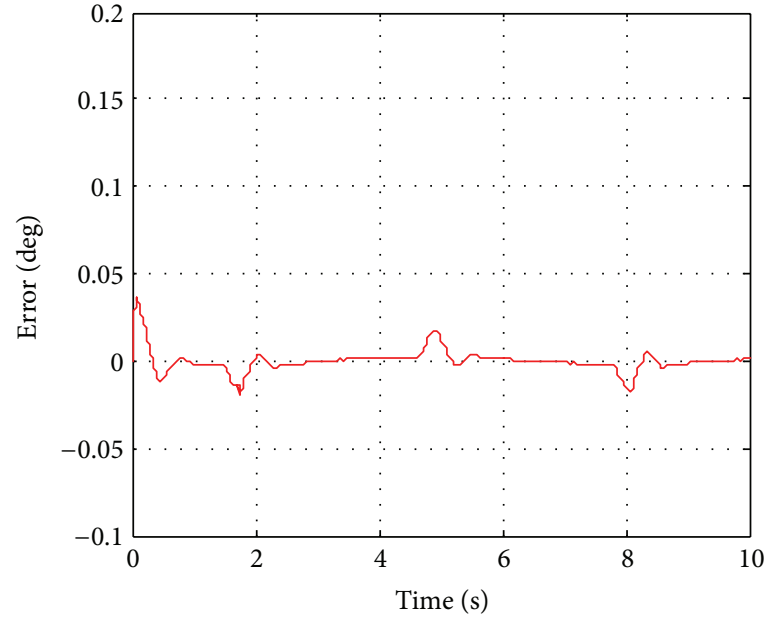

(b) Tracking error

FIGURE 12: Simulation results for the $0.6^{\circ} / \mathrm{s}$ and $0.6^{\circ} / \mathrm{s}^{2}$ sine signal input.

We get

$$
\begin{aligned}
Q_{11}(k) & Q_{33}(k)-Q_{13}^{2}(k) \\
= & -\left[p_{13}(k)+a_{13}(k+1)\right]^{2}+Q_{11}(k) Q_{33}(k) \\
= & -\left[p_{13}(k)-p_{13}(k+1)\right]^{2} \\
& +S_{13}\left[p_{13}(k)-p_{13}(k+1)\right]+T_{13},
\end{aligned}
$$

where

$$
\begin{aligned}
S_{13}= & -2 p_{13}(k+1)-2 a_{13}(k+1), \\
T_{13}= & p_{13}^{2}(k+1)+S_{13} p_{13}(k+1) \\
& -a_{13}^{2}(k+1)+Q_{11}(k) Q_{33}(k) .
\end{aligned}
$$




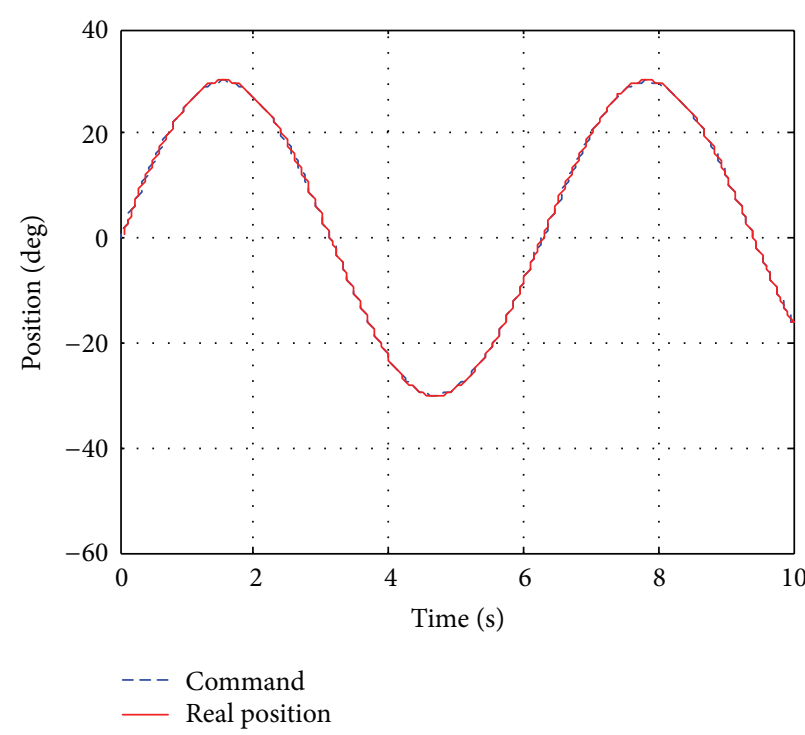

(a) Position tracking response

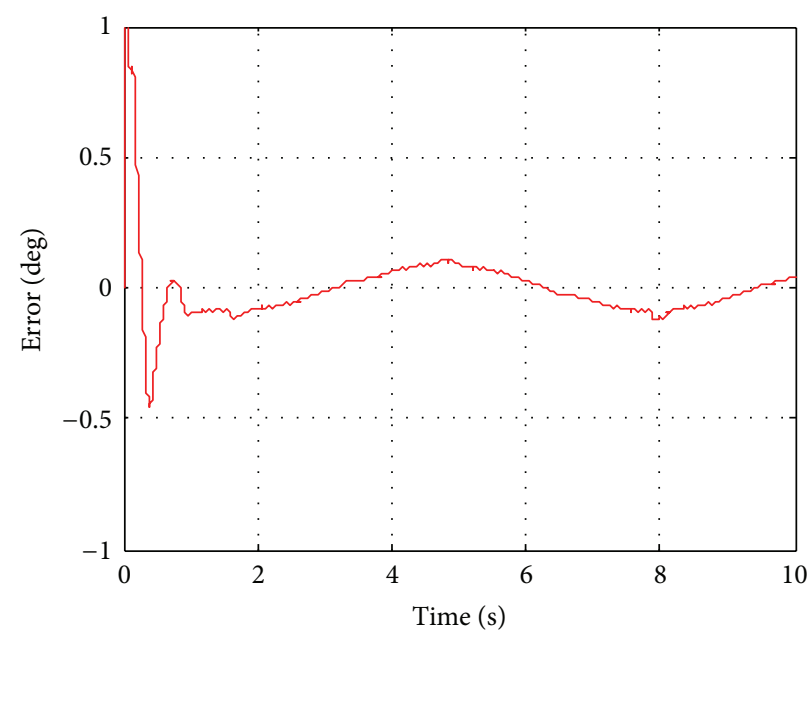

(b) Tracking error

FIGURE 13: Simulation results for the $30^{\circ} / \mathrm{s}$ and $30^{\circ} / \mathrm{s}^{2}$ sine signal input.

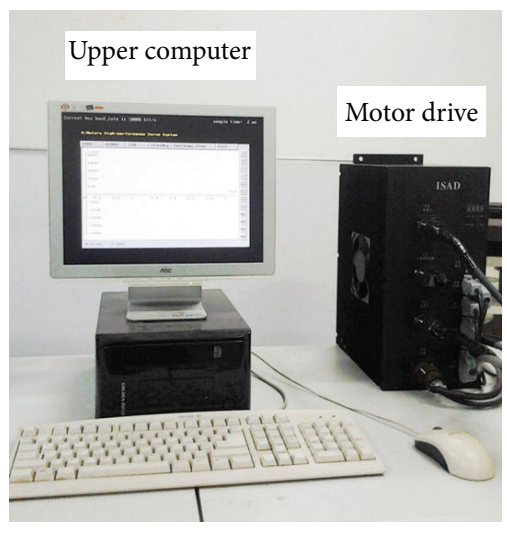

(a) Upper computer and servo drive

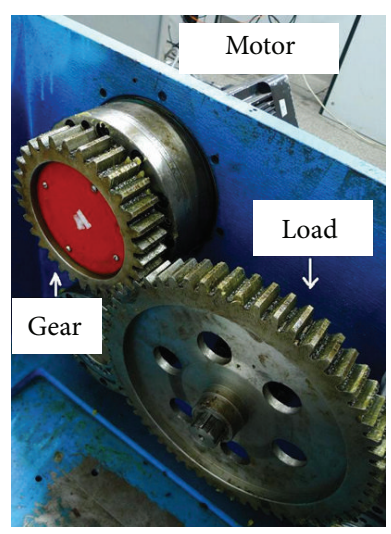

(b) Motor and load

FIgURE 14: Experimental system.

$S_{13}$ and $T_{13}$ can be expressed in the form of (29) and (28):

$$
\begin{array}{cc}
B(k+1)=p_{13}(k+1), & C(k+1)=a_{13}(k+1), \\
D(k)=Q_{11}(k) Q_{33}(k), & F(k)=0,
\end{array}
$$

Then the discriminant root of the quadratic trinomial (64) about $\left[p_{13}(k)-p_{13}(k+1)\right]$ is

$$
\begin{aligned}
\Delta_{13}(k) & =F^{2}(k)+4 M(k) D(k) \\
& =4 Q_{11}(k) Q_{33}(k)=4 M_{\mathrm{Q} 1}(k) Q_{33}(k),
\end{aligned}
$$

where

$$
Q_{33}(k)=p_{33}-p_{22}+2 \varepsilon_{1} f_{1}^{2}(k+1)-p_{33} f_{1}^{2}(k+1) .
$$

We can get $Q_{33}(k)>0$ by $(44)$, so $\Delta_{13}(k)>0$. According to (40) and Lemma 11, we obtain

$$
Q_{11}(k) Q_{33}(k)-Q_{13}^{2}(k)>0 \text {. }
$$

So we get $\Delta_{23}(k)>0$ by $(62)$. Then we have $M_{\mathrm{Q} 3}(k)>0$, according to (39) and Lemma 11.

Thus, $Q(k)$ is a uniformly bounded, positive definite symmetric matrix by Lemma 10. Furthermore, the timevarying discrete system (22) is uniformly asymptotically stable by Lemma 9. The proof is completed.

\section{Simulation Results}

Considering the characters of the servo system, a composite controller which is composed of golden-section adaptive control law, integral control law, and feed-forward control 


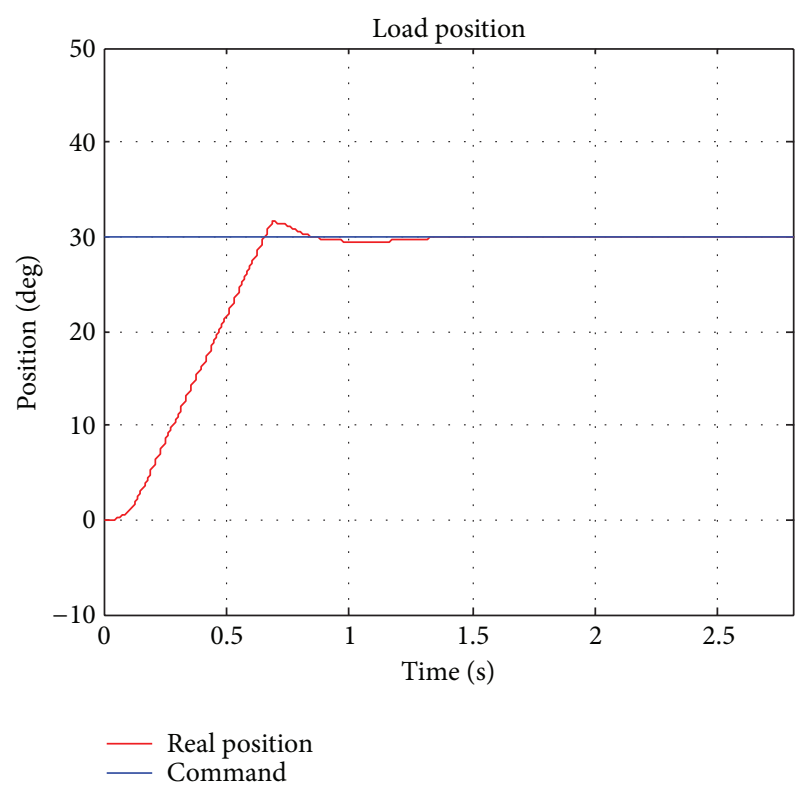

(a) Position tracking response (PIF)

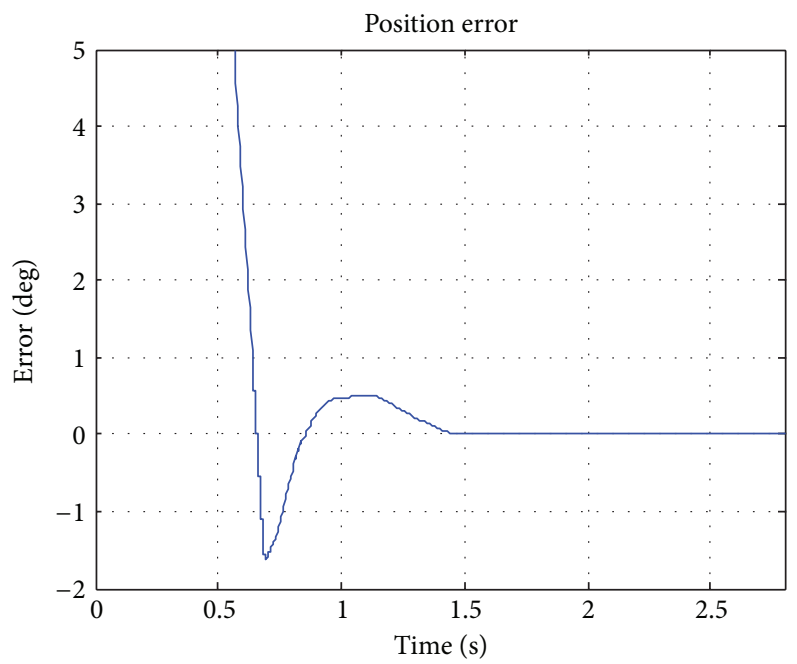

(c) Tracking error (PIF)

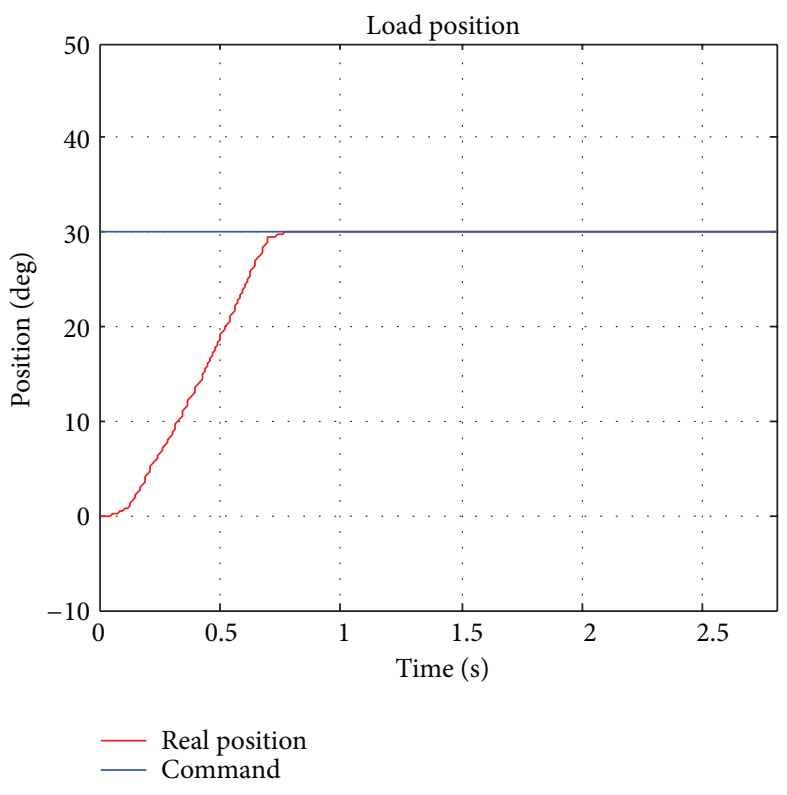

(b) Position tracking response (GSAIF)

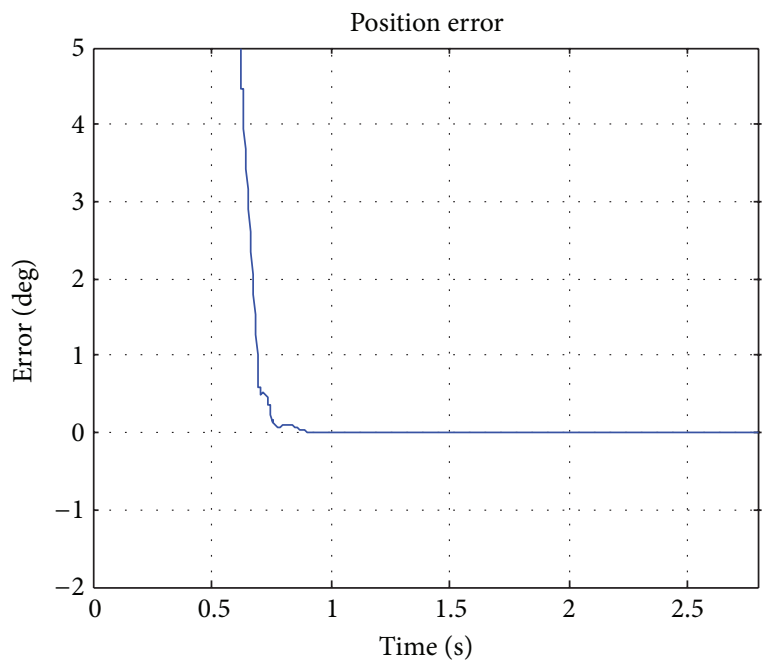

(d) Tracking error (GSAIF)

FIGURE $15: 30^{\circ}$ step response.

law is applied in the system simulation. The structure of the closed-loop system is shown in Figure 8.

The golden-section adaptive control law $u_{l}(k)$ is given as

$$
u_{l}(k)=\frac{1}{\widehat{\beta}_{0}(k)+\lambda}\left[l_{1} \widehat{\alpha}_{1}(k) e(k)+l_{2} \widehat{\alpha}_{2}(k) e(k-1)\right],
$$

where $e(k)=y_{r}(k)-y(k), l_{1}=0.382, l_{2}=0.618$, and $\lambda$ is a small positive constant which ensures that the golden-section adaptive control law is bounded when $\beta_{0}(k)=0$.

The integral control law is given as

$$
u_{i}(k)=u_{i}(k-1)+k_{i} e(k) .
$$

The feed-forward control law is given as

$$
u_{f}(k)=k_{f}\left(y_{r}(k)-y_{r}(k-1)\right),
$$

where $k_{f}$ is the feed-forward coefficient.
In summary, the composite controller is

$$
u(k)=u_{a}(k)+u_{f}(k)=u_{l}(k)+u_{i}(k)+u_{f}(k) .
$$

The values of the controller parameters are $\lambda=2 \times$ $10^{-3}, k_{i}=0.6$, and $k_{f}=50$.

In order to verify the effectiveness of the servo system, step signal, slope signal, and sine signal, which are often used in the performance test of servo systems, are used as input signals in this paper. In the simulations, the system responses under $30^{\circ}$ step signal input, $0.6^{\circ} / \mathrm{s}$ slope signal input, $30^{\circ} / \mathrm{s} \mathrm{slope} \mathrm{signal} \mathrm{input,} 0.6^{\circ} / \mathrm{s}, 0.6^{\circ} / \mathrm{s}^{2}$ sine signal input, and $30^{\circ} / \mathrm{s}, 30^{\circ} / \mathrm{s}^{2}$ sine signal input are tested, respectively.

(1) When the input signal is $y_{r}(t)=30^{\circ}$, the results are shown in Figure 9. 


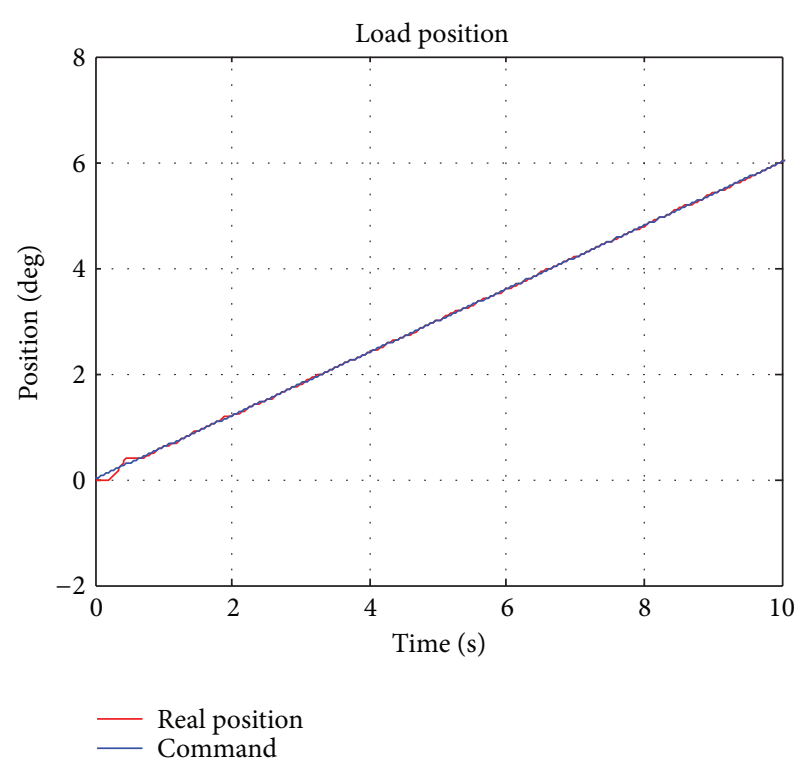

(a) Position tracking response (PIF)

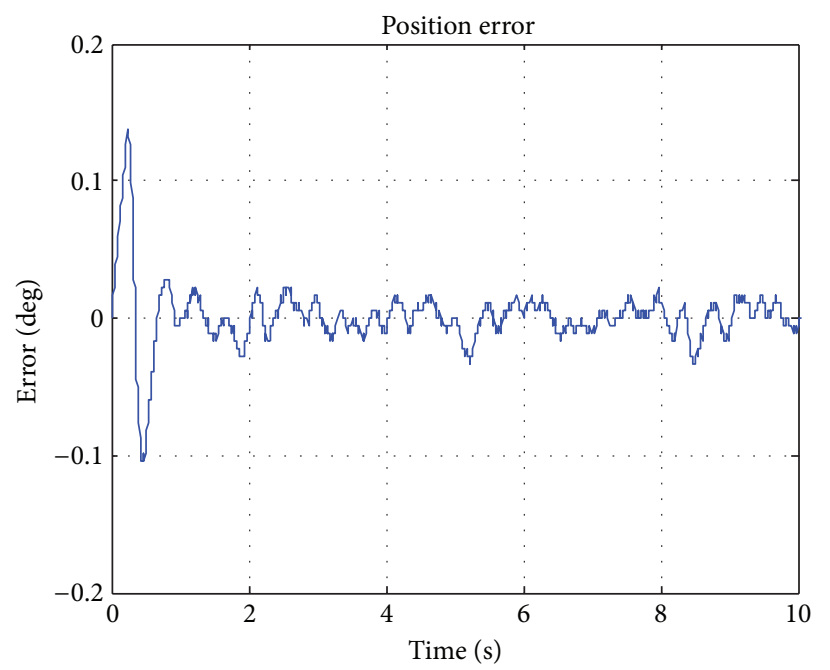

(c) Tracking error (PIF)

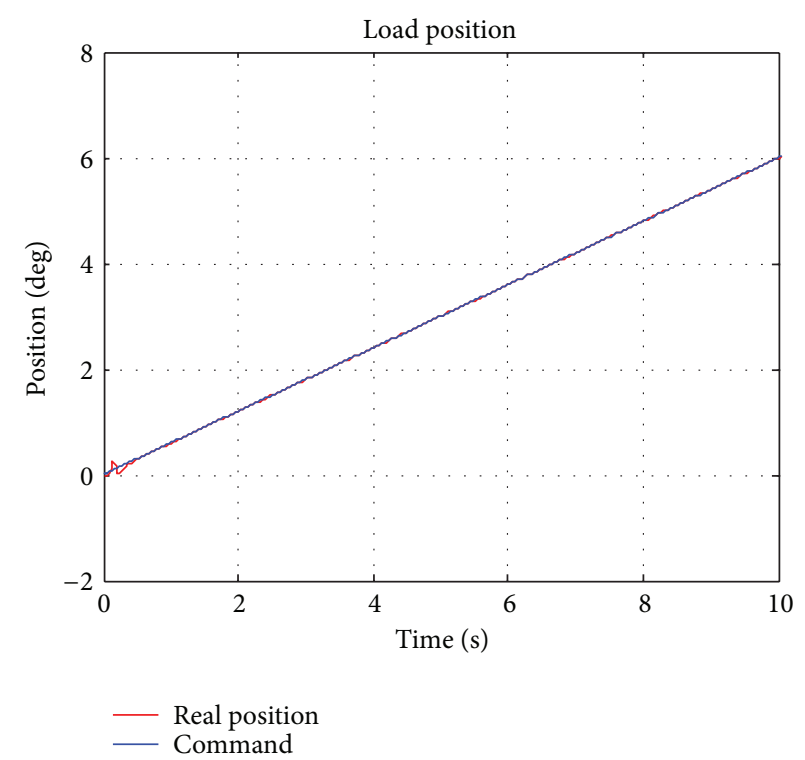

(b) Position tracking response (GSAIF)

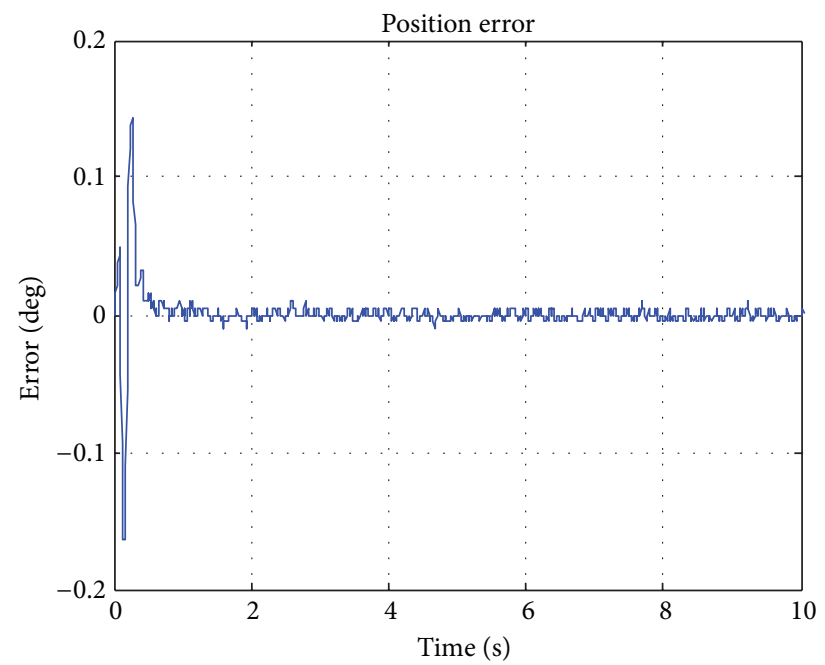

(d) Tracking error (GSAIF)

FIGURE 16: $0.6^{\circ} / \mathrm{s}$ slope response.

(2) When the input signal is $y_{r}(t)=0.6^{\circ} / \mathrm{s}$, the results are shown in Figure 10.

(3) When the input signal is $y_{r}(t)=30^{\circ} / \mathrm{s}$, the results are shown in Figure 11.

(4) When the input signal is $y_{r}(t)=0.6^{\circ} \sin (t)$, the results are shown in Figure 12.

(5) When the input signal is $y_{r}(t)=30^{\circ} \sin (t)$, the results are shown in Figure 13.

\section{Experimental Results}

To verify the effectiveness of the proposed control scheme, the experiments are conducted on the experimental system as shown in Figure 14. The experimental system consisted of an upper computer, a servo drive, a servo motor, a reducer, and a load. The PC104 (SCM/SPT4) module is used as the upper computer, and it is mainly used for sending instructions and monitoring the status of the system. The servo drive is designed by us based on the DSP TMS320F28335, which has a CAN communication interface and can receive the position instruction from the upper computer in real time. In the running process, the drive implements the control of current, 


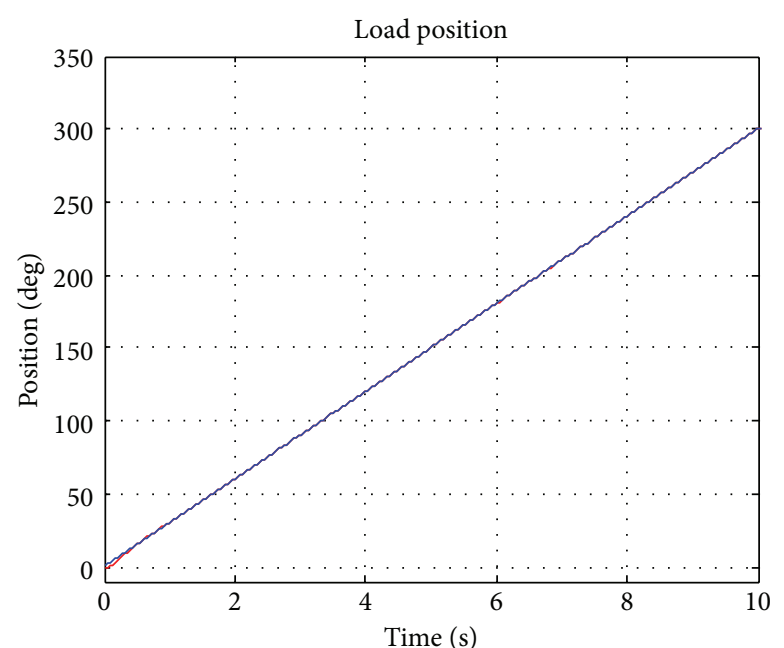

- Real position

(a) Position tracking response (PIF)

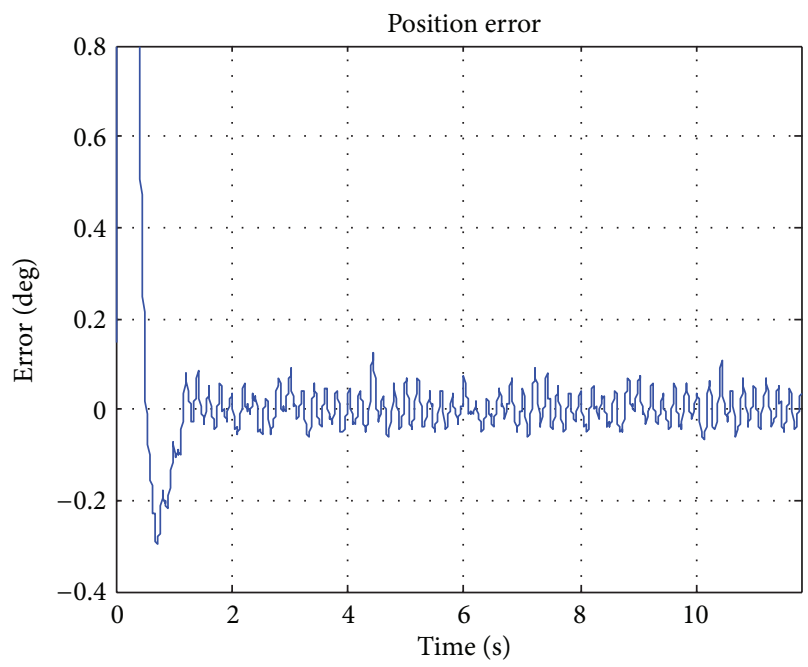

(c) Tracking error (PIF)

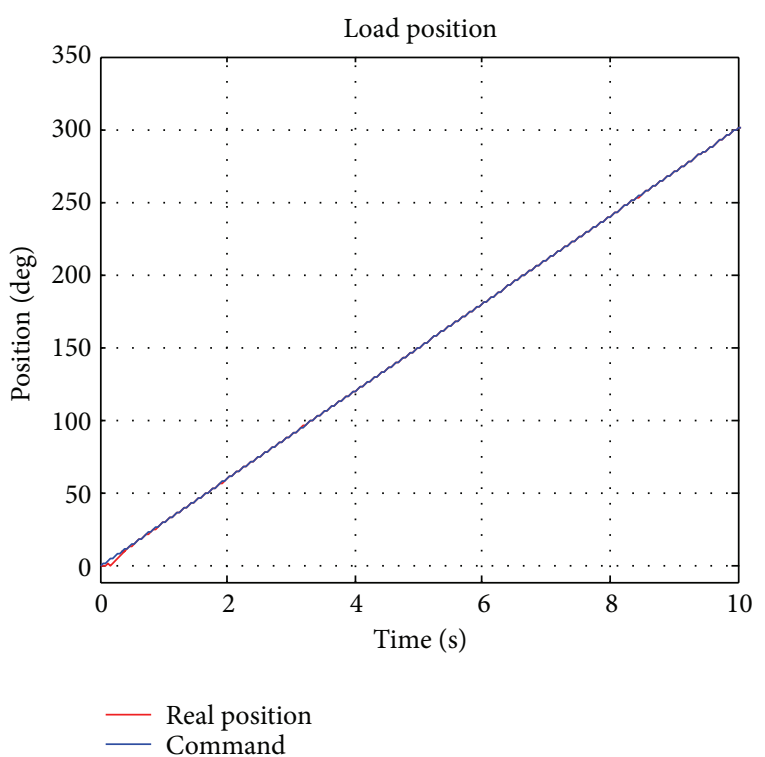

(b) Position tracking response (GSAIF)

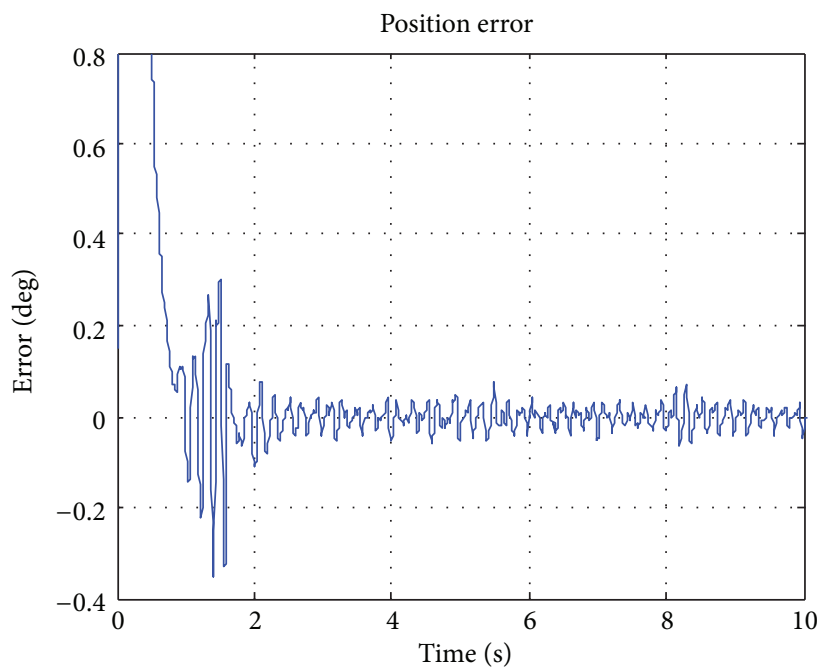

(d) Tracking error (GSAIF)

FIGURE $17: 30^{\circ} / \mathrm{s}$ slope response.

speed, and position of the motor. The PMSM (M-403-B) produced by Kollmorgen Company is selected as the servo motor in the experiment. The parameters of the motor are listed in Table 1. The high speed and high rigidity planetary gear reducer (FIC-A35-119) produced by Sumitomo Heavy Machinery Company is utilized in the experiment, whose reduction ratio is $1: 119$, and the reduction ratio of the final gears is $1: 2$.

The backlash between the motor and the load is $0.36^{\circ}$. PI control scheme is used in the speed controller and current controller of the motor drive; the proportion and integral gains of the speed controller and current controller are 1.2, $0.02,1.5$, and 0.02 , respectively, after adjustment. For the sake of comparison, the position controller based on PI control scheme with feed-forward compensation (PIF) and the composite position controller including the golden-section
TABle 1: Parameters of the servo motor (M-403-B)

\begin{tabular}{lcc}
\hline Name & Units & Value \\
\hline Rated power & $\mathrm{kW}$ & 2.5 \\
Rated speed & $\mathrm{rpm}$ & 3000 \\
Torque coefficient & $\mathrm{N} \cdot \mathrm{m} / \mathrm{A}$ & 1.11 \\
EMF & $\mathrm{V} / \mathrm{krpm}$ & 67.2 \\
Line resistance & $\mathrm{ohms}$ & 2.6 \\
Line induction & $\mathrm{mH}$ & 50 \\
Inertia & $\mathrm{Kg} \cdot \mathrm{m}^{2}$ & 0.00259 \\
Static friction & $\mathrm{N} \cdot \mathrm{m}$ & 0.24 \\
Viscous friction & $\mathrm{N} \cdot \mathrm{m} / \mathrm{krpm}$ & 0.015 \\
\hline
\end{tabular}

adaptive control law, the integral control law, and feed forward compensation (GSAIF) are tested, respectively, in the 


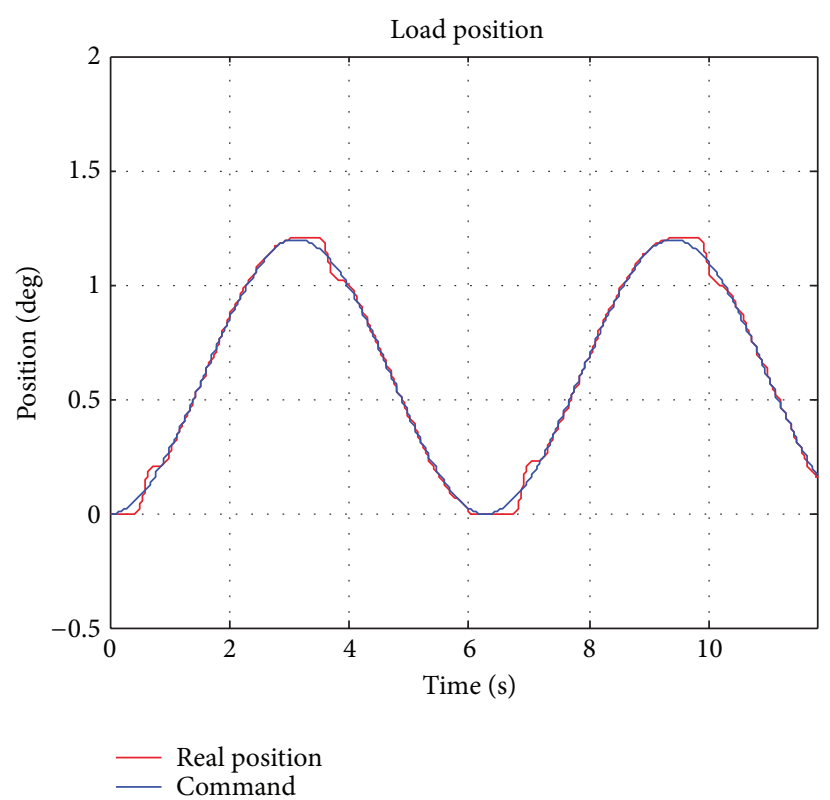

(a) Position tracking response (PIF)

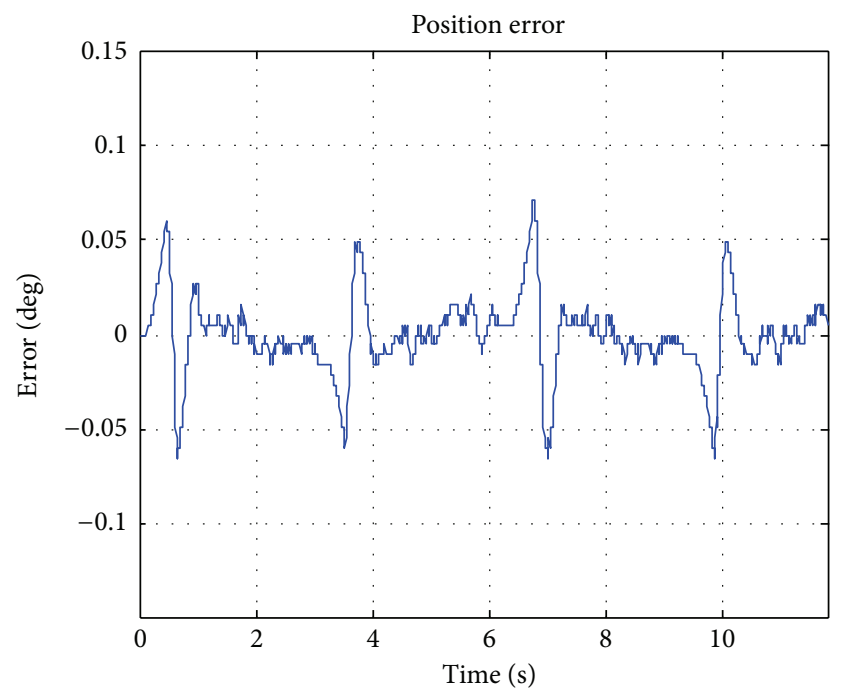

(c) Tracking error (PIF)

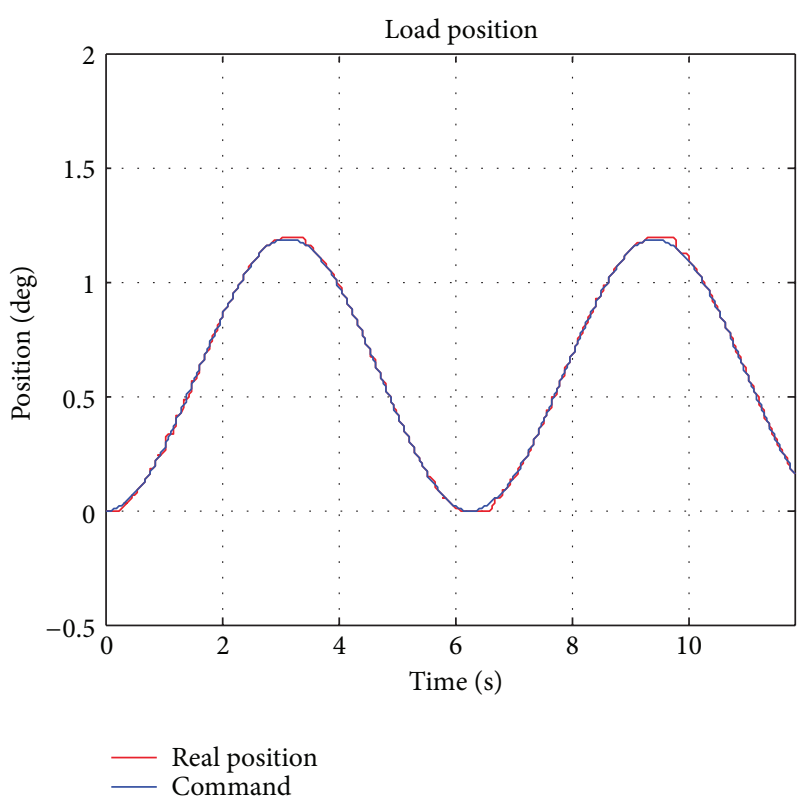

(b) Position tracking response (GSAIF)

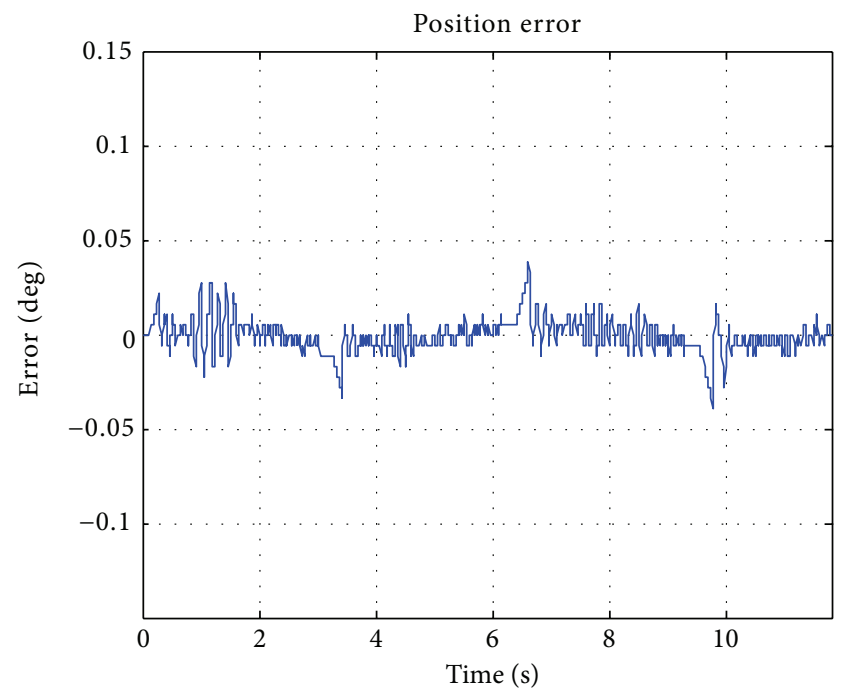

(d) Tracking error (GSAIF)

FIgURE 18: $0.6^{\circ} / \mathrm{s}$ and $0.6^{\circ} / \mathrm{s}^{2}$ sine response.

experiment. In the PIF scheme, the proportion and integral gains are 4.2 and 0.23 , respectively, and the feed-forward gain is 0.25 . In the GSAIF scheme, $f=0.995$, and $\lambda=$ 0.01 , the coefficients of integral gain and feed forward gain are 0.29 and 0.25 . Position step, slope, and sine signals are used in the experiments to test the system performance. The experimental results are shown in Figures 15-19.

(1) Tracking the $30^{\circ}$ Step Signal. From Figure 15 it can be obtained that the system overshoot is $1.8^{\circ}$ using PIF control scheme; after $1.5 \mathrm{~s}$ the system is in a stable state and the tracking error is zero. When using GSAIF control scheme, the overshoot does not appear, and after $0.8 \mathrm{~s}$ the system is in a stable state and the stable tracking error is zero too. The comparison results show that the GSAIF scheme has a better dynamic performance and the response speed is much better than the PIF scheme.

(2) Tracking the $0.6 \%$ and the $30 \%$ s Slope Signal. As shown in Figures 16 and 17, the system is tested for tracking $0.6^{\circ} / \mathrm{s}$ and $30^{\circ} / \mathrm{s}$ position slope signal. In the $0.6^{\circ} / \mathrm{s}$ tracking experiment, the maximum stable error is $0.023^{\circ}$ using PIF scheme. When the system tracks the command signal using GSAIF, the maximum tracking error is only $0.01^{\circ}$. In the $30 \%$ tracking test, the stable error increases using both of the control schemes. The maximum stable tracking error 


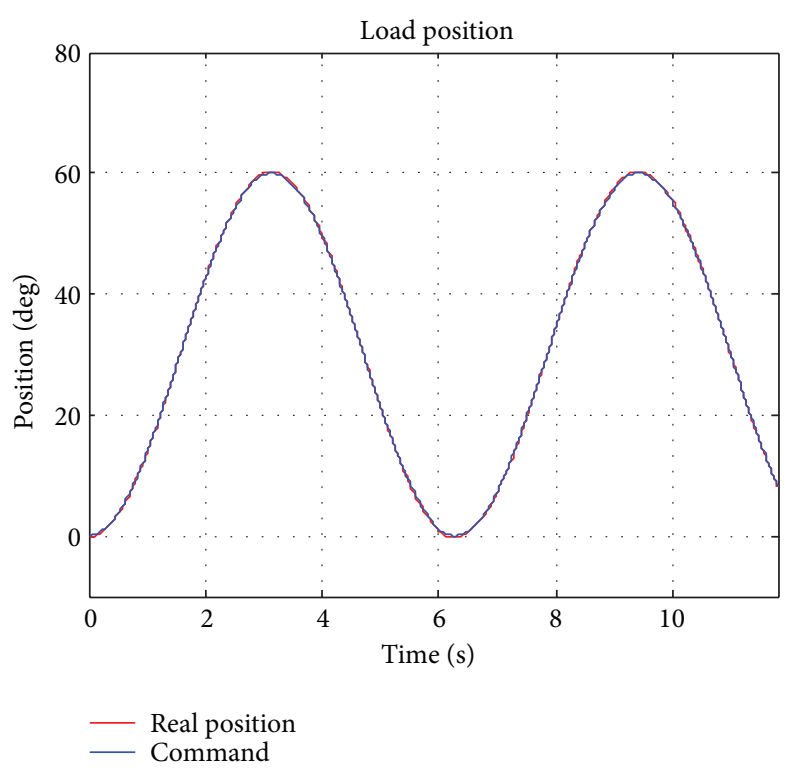

(a) Position tracking response (PIF)

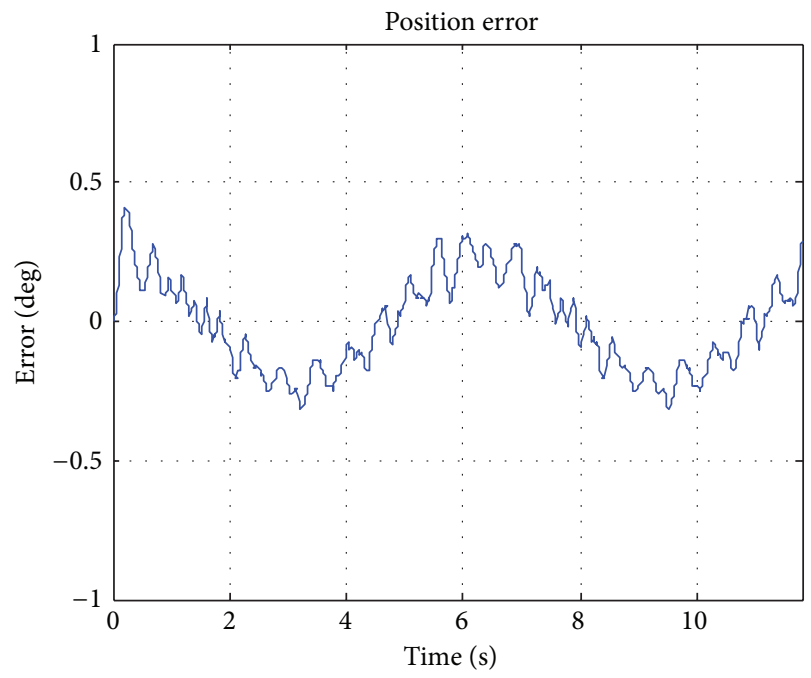

(c) Tracking error (PIF)

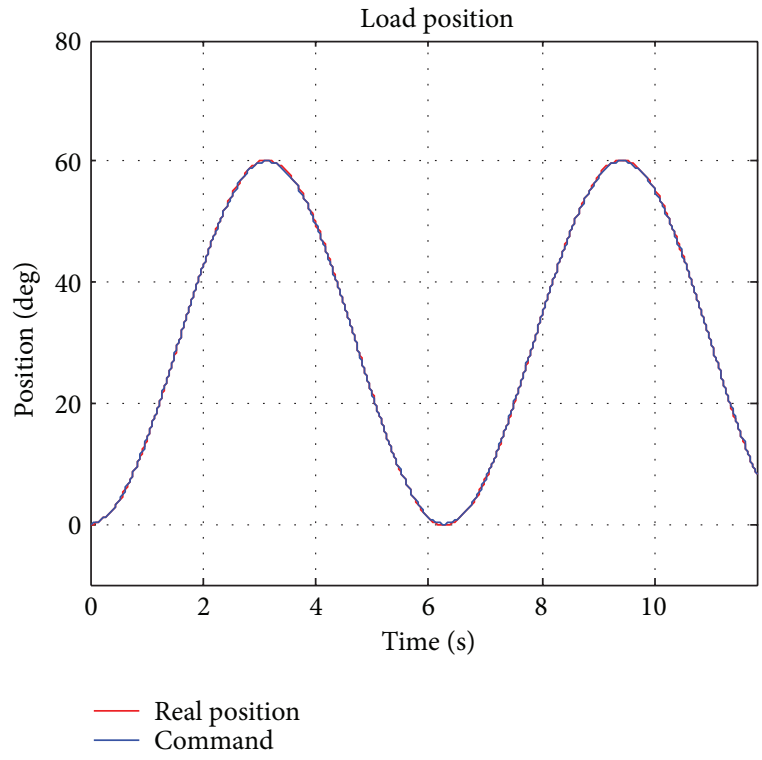

(b) Position tracking response (GSAIF)

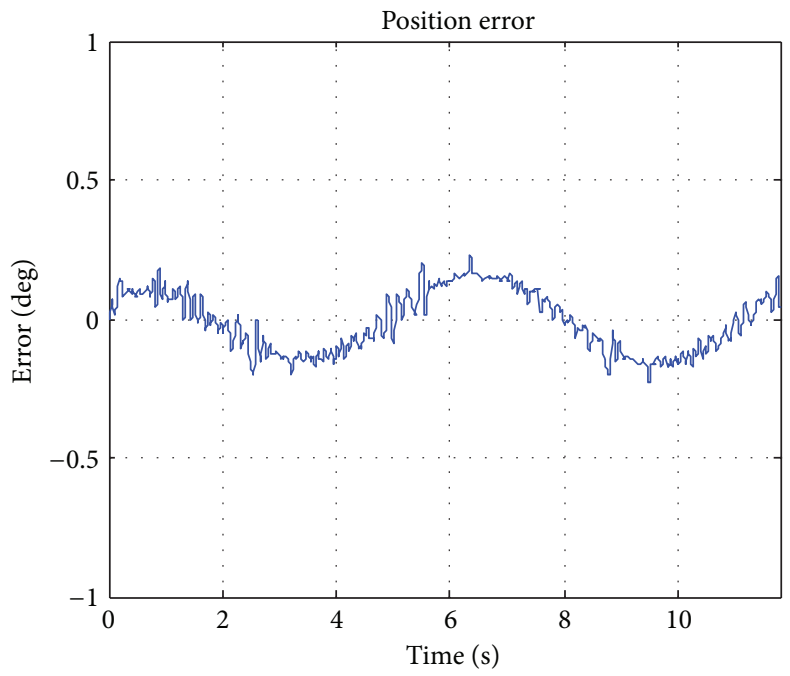

(d) Tracking error (GSAIF)

FigURE 19: $30 \%$ and $30 \% \mathrm{~s}^{2}$ sine response.

using PIF scheme is $0.12^{\circ}$. When using GSAIF scheme, the maximum tracking error is $0.08^{\circ}$. The comparison results show that the GSAIF scheme has better smoothness and higher precision tracking performance compared with PIF scheme when tracking the position slope signals.

(3) Tracking the $0.6^{\circ} / \mathrm{s}, 0.6^{\circ} / \mathrm{s}^{2}$ and $30^{\circ} / \mathrm{s}, 30^{\circ} / \mathrm{s}^{2}$ Sine Signal. As shown in Figures 18 and 19, the system is tested for tracking position sine signal; the maximum speed and acceleration of the command signals are $0.6^{\circ} / \mathrm{s}, 0.6^{\circ} / \mathrm{s}^{2}$ and $30^{\circ} / \mathrm{s}, 30^{\circ} / \mathrm{s}^{2}$, respectively. In the $0.6^{\circ} / \mathrm{s}, 0.6^{\circ} / \mathrm{s}^{2}$ sine signal tracking test, the maximum tracking error is $0.06^{\circ}$ when using PIF scheme. However, using GSAIF scheme, the maximum tracking error is $0.04^{\circ}$. In the $30^{\circ} / \mathrm{s}, 30^{\circ} / \mathrm{s}^{2}$ sine signal tracking test, the tracking error vibrates severely using the PIF scheme and the maximum tracking error reaches $0.42^{\circ}$. Using GSAIF scheme, there is no obvious vibrations and the maximum tracking error is only $0.26^{\circ}$. The comparison results show that the GSAIF scheme possesses a better suppression ability of nonlinear uncertainties such as backlash and friction when tracking sine signals, and it improves the system tracking precision.

\section{Conclusions}

In this paper, the characteristic modeling technique is applied for modeling and control of the servo system with backlash and friction. To meet the demands of characteristic modeling, 
a continuous approximation of dead-zone function is introduced to describe the backlash model, and the $\operatorname{sgn}(\cdot)$ function is replaced by $(2 / \pi) \arctan (k v)$ to make the Stribeck friction model smooth. The characteristic model of the system is established based on a second-order slowly time-varying difference equation and verified by simulations. The composite controller based on golden-section adaptive law, feedforward compensation, and integral law is proposed, and the stability of the closed-loop system is analyzed by Lyapunov theory. It is shown by both simulation and experimental results that it is feasible to establish the characteristic model of the servo system with backlash and friction. The GSAIF controller is also effective, and it can reduce the effect of backlash and friction and improve the steady-state precision and the dynamic performance of the servo system.

\section{Conflict of Interests}

The authors declare that there is no conflict of interests regarding the publication of this paper.

\section{Acknowledgment}

This work was supported by the National Natural Science Foundation of China under Grant nos. 61074023 and 60974027.

\section{References}

[1] B. Armstrong-Hélouvry, P. Dupont, and C. C. de Wit, "A survey of models, analysis tools and compensation methods for the control of machines with friction," Automatica, vol. 30, no. 7, pp. 1083-1138, 1994.

[2] M. Nordin and P.-O. Gutman, "Controlling mechanical systems with backlash-a survey," Automatica, vol. 38, no. 10, pp. 16331649, 2002.

[3] G. Tao and P. V. Kokotović, "Continuous-time adaptive control of systems with unknown backlash," IEEE Transactions on Automatic Control, vol. 40, no. 6, pp. 1083-1087, 1995.

[4] R. R. Selmic and F. L. Lewis, "Neural net backlash compensation with Hebbian tuning using dynamic inversion," Automatica, vol. 37, no. 8, pp. 1269-1277, 2001.

[5] G. Tao, X. Ma, and Y. Ling, "Optimal and nonlinear decoupling control of systems with sandwiched backlash," Automatica, vol. 37, no. 2, pp. 165-176, 2001.

[6] J. Zhou, C. Wen, and Y. Zhang, "Adaptive output control of nonlinear systems with uncertain dead-zone nonlinearity," IEEE Transactions on Automatic Control, vol. 51, no. 3, pp. 504511, 2006.

[7] P. Rostalski, T. Besselmann, M. Barić, F. van Belzen, and M. Morari, "A hybrid approach to modelling, control and state estimation of mechanical systems with backlash," International Journal of Control, vol. 80, no. 11, pp. 1729-1740, 2007.

[8] R. Merzouki, J. A. Davila, L. Fridman, and J. C. Cadiou, "Backlash phenomenon observation and identification in electromechanical system," Control Engineering Practice, vol. 15, no. 4, pp. 447-457, 2007.

[9] A. Lagerberg and B. Egardt, "Backlash estimation with application to automotive powertrains," IEEE Transactions on Control Systems Technology, vol. 15, no. 3, pp. 483-493, 2007.
[10] S. I. Han and J. M. Lee, "Adaptive dynamic surface control with sliding mode control and RWNN for robust positioning of a linear motion stage," Mechatronics, vol. 22, no. 2, pp. 222-238, 2012.

[11] Y.-T. Liu, T.-T. Kung, K.-M. Chang, and S.-Y. Chen, "Observerbased adaptive sliding mode control for pneumatic servo system," Precision Engineering, vol. 37, no. 3, pp. 522-530.

[12] B. A. Bucci, J. S. Vipperman, D. G. Cole, and S. J. Ludwick, "Evaluation of a servo settling algorithm," Precision Engineering, vol. 37, no. 1, pp. 10-22, 2013.

[13] C. Canudas de Wit, H. Olsson, K. J. Åström, and P. Lischinsky, "A new model for control of systems with friction," IEEE Transactions on Automatic Control, vol. 40, no. 3, pp. 419-425, 1995.

[14] L. Márton and B. Lantos, "Control of mechanical systems with Stribeck friction and backlash," Systems \& Control Letters, vol. 58, no. 2, pp. 141-147, 2009.

[15] B. Bona and M. Indri, "Friction compensation in robotics: an overview," in Proceedings of the 44th IEEE Conference on Decision and Control, and the European Control Conference (CDC-ECC '05), pp. 4360-4367, Seville, Spain, December 2005.

[16] B. Armstrong, D. Neevel, and T. Kusik, "New results in NPID control: tracking, integral control, friction compensation and experimental results," IEEE Transactions on Control Systems Technology, vol. 9, no. 2, pp. 399-406, 2001.

[17] D. Garagić and K. Srinivasan, "Adaptive friction compensation for precision machine tool drive," Control Engineering Practice, vol. 12, no. 11, pp. 1451-1464, 2004.

[18] H. Chaoui and P. Sicard, "Adaptive fuzzy logic control of permanent magnet synchronous machines with nonlinear friction," IEEE Transactions on Industrial Electronics, vol. 59, no. 2, pp. 1123-1133, 2012.

[19] Y. Wang, D. Wang, and T. Chai, "Extraction and adaptation of fuzzy rules for friction modeling and control compensation," IEEE Transactions on Fuzzy Systems, vol. 19, no. 4, pp. 682-693, 2011.

[20] S. N. Huang, K. K. Tan, and T. H. Lee, "Adaptive motion control using neural network approximations," Automatica, vol. 38, no. 2, pp. 227-233, 2002.

[21] S.-W. Lee, "Robust adaptive stick slip compensation," Industrial Electronics, vol. 22, no. 5, pp. 747-779, 1995.

[22] H. Xu and P. A. Ioannou, "Robust adaptive control of linearizable nonlinear single input systems with guaranteed error bounds," Automatica, vol. 40, no. 11, pp. 1905-1911, 2004.

[23] K. Menon and K. Krishnamurthy, "Control of low velocity friction and gear backlash in a machine tool feed drive system," Mechatronics, vol. 9, no. 1, pp. 33-52, 1999.

[24] S. Suraneni, I. N. Kar, O. V. R. Murthy, and R. K. P. Bhatt, "Adaptive stick-slip friction and backlash compensation using dynamic fuzzy logic system," Applied Soft Computing Journal, vol. 6, no. 1, pp. 26-37, 2005.

[25] H. Wu, J. Hu, and Y. Xie, "Characteristic model-based allcoefficient adaptive control method and its applications," IEEE Transactions on Systems, Man and Cybernetics C, vol. 37, no. 2, pp. 213-221, 2007.

[26] B. Meng and H.-X. Wu, "A unified proof of the characteristic model of linear time-invariant systems," in Proceedings of the American Control Conference (ACC '07), pp. 935-940, New York, NY, USA, July 2007.

[27] Y. Wang, "Stability analysis of characteristic model based allcoefficient adaptive control for a class of minimum-phase linear system," Procedia Engineering, vol. 29, pp. 2410-2420, 2012. 
[28] H. Wu, J. Hu, and Y. Xie, Characteristic Model-Based Intelligent Adaptive Control, Chinese Science and Technology Press, Beijing, China, 2008.

[29] R.-H. Du, Y.-F. Wu, W. Chen, and Q.-W. Chen, "Adaptive backstepping fuzzy control for servo systems with backlash," Control Theory and Applications, vol. 30, no. 2, pp. 254-260, 2013.

[30] D.-Q. Sun and H.-X. Wu, "Uniform asymptotic stability of 3rdorder time-variant discrete systems," Journal of Astronautics, vol. 25, no. 5, pp. 502-506, 2004. 


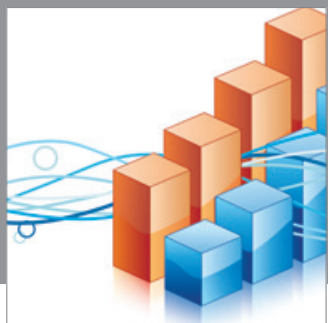

Advances in

Operations Research

mansans

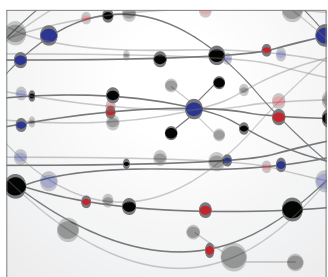

The Scientific World Journal
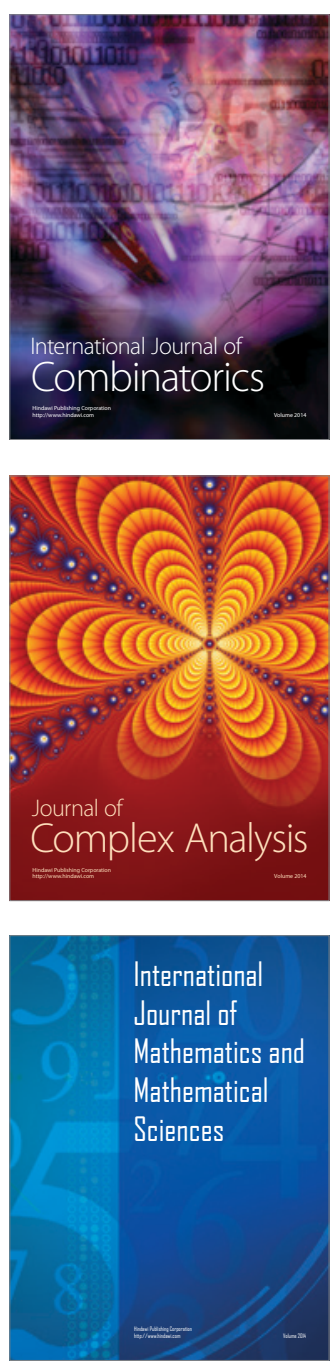
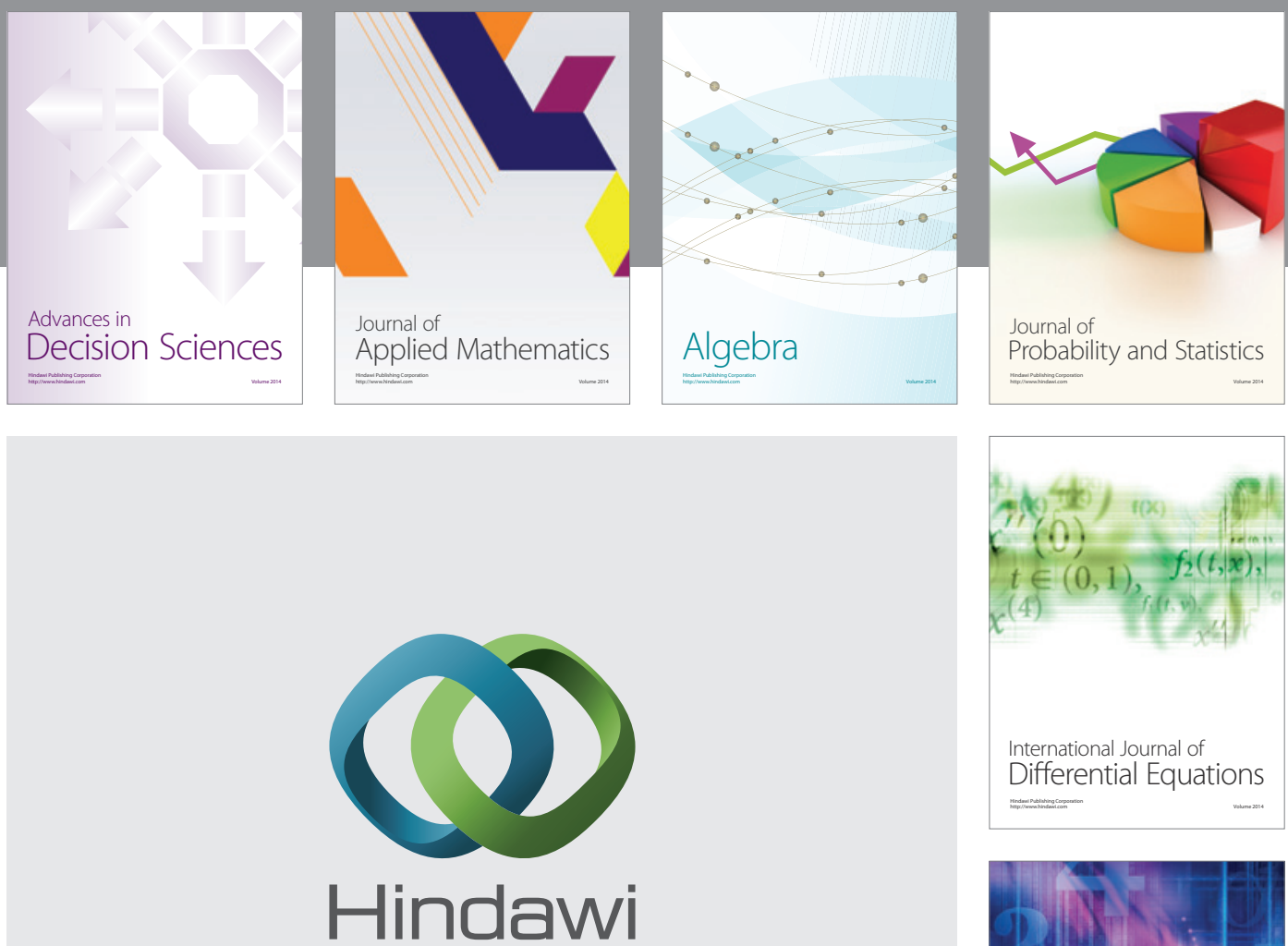

Submit your manuscripts at http://www.hindawi.com
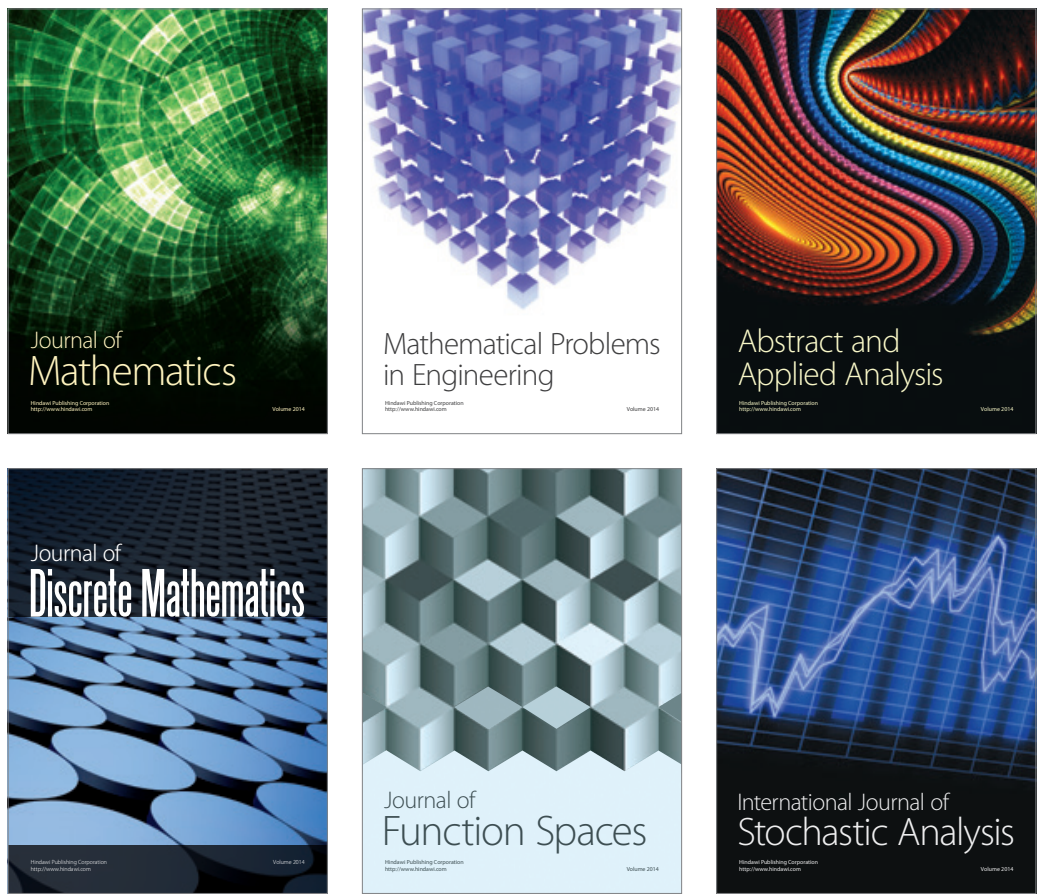

Journal of

Function Spaces

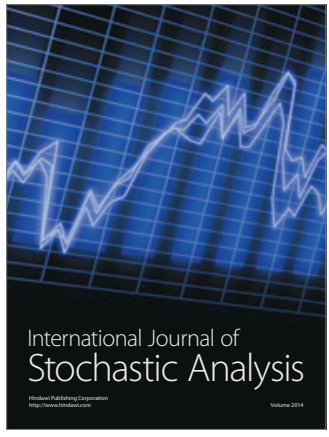

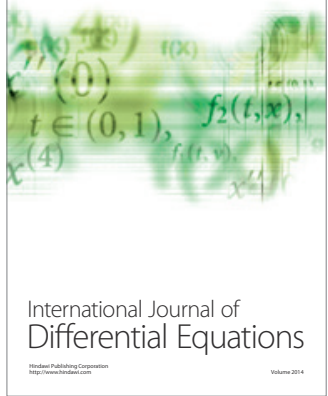
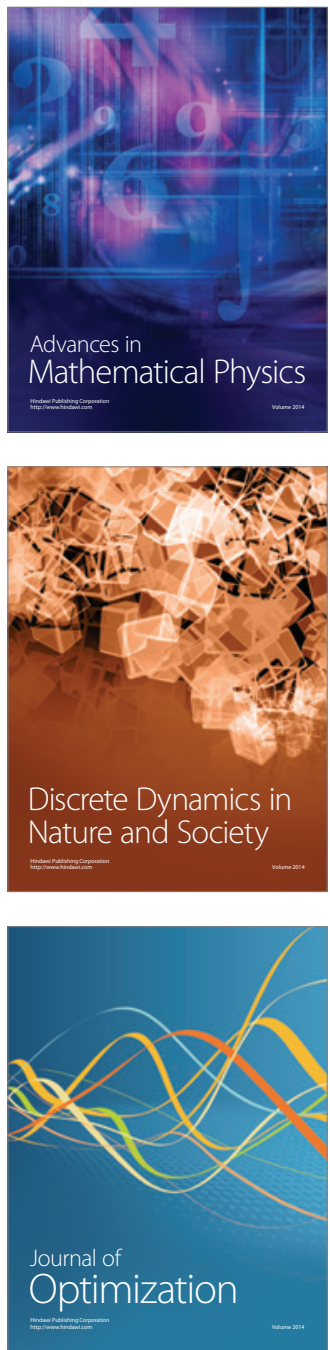